\title{
The energy of waves in the photosphere and lower chromosphere
}

\section{Inversion setup for Ca II H spectra in local thermal equilibrium ${ }^{\star}$}

\author{
C. Beck ${ }^{1,2}$, R. Rezaei ${ }^{3}$, and K. G. Puschmann ${ }^{4}$ \\ ${ }^{1}$ Instituto de Astrofísica de Canarias (IAC), via Lactéa, 38205 La Laguna (Tenerife), Spain \\ e-mail: cbeck@iac.es \\ 2 Departamento de Astrofísica, Universidad de La Laguna, 38206 La Laguna (Tenerife), Spain \\ 3 Kiepenheuer-Institut für Sonnenphysik (KIS), Schöneckstr. 6, 79104 Freiburg, Germany \\ e-mail: rrezaei@kis .uni-freiburg.de \\ ${ }^{4}$ Leibniz-Institut für Astrophysik Potsdam (AIP), An der Sternwarte 16, 14482 Potsdam, Germany \\ e-mail: kgp@aip.de
}

Received 18 July 2012 / Accepted 27 September 2012

\section{ABSTRACT}

\begin{abstract}
Context. The CaII H line is one of the strongest lines in the solar spectrum, and it provides continuous information on the solar atmosphere from the photosphere to the lower chromosphere.

Aims. We describe an inversion approach that reproduces observed Ca II H spectra by assuming local thermal equilibrium (LTE). Methods. We developed an inversion strategy based on the SIR code that reproduces Ca II H spectra in the LTE approximation. The approach uses a two-step procedure with an archive of pre-calculated spectra to fit the line core and a subsequent iterative modification to improve the fit mainly in the line wing. Simultaneous spectra in the $630 \mathrm{~nm}$ range can optionally be used to fix the continuum temperature. The method retrieves one-dimensional (1D) temperature stratifications while neglecting lateral radiative transport. Lineof-sight velocities are included post facto with an empirical approach.

Results. An archive of about 300000 pre-calculated spectra is more than sufficient to reproduce the line core of observed Ca II $\mathrm{H}$ spectra both in the quiet Sun and in active regions. The subsequent iterative adjustment of the thermodynamical stratification matches observed and best-fit spectra to a level of about $0.5 \%$ of $I_{\mathrm{c}}$ in the line wing and about $1 \%$ of $I_{\mathrm{c}}$ in the line core.

Conclusions. The successful application of the LTE inversion strategy suggests that inversion schemes based on pre-calculated spectra allow a reliable and relatively fast retrieval of solar properties from observed chromospheric spectra. The approach can be easily extended to a 1D non-LTE (NLTE) case by a simple exchange of the pre-calculated archive spectra. Using synthetic NLTE spectra from numerical three-dimensional (3D) simulations instead will finally allow one to extend the approach from the static 1D-case to dynamical atmosphere models, including the complete 3D radiative transport.
\end{abstract}

Key words. Sun: chromosphere - Sun: oscillations

\section{Introduction}

The spectral lines of Ca II H at $396.8 \mathrm{~nm}$ and Ca II K at $393.3 \mathrm{~nm}$ are among the strongest and deepest spectral lines in the visible solar spectrum. The intensity radiated away inside these lines comes from layers between the continuum forming layer and the lower chromosphere, spanning a range of about 1-2 Mm of height in the solar atmosphere (Vernazza et al. 1981; Carlsson $\&$ Stein 1997). Both lines have thus been used intensively for studies of the chromosphere, where useful spectral lines are scarce. Ground-based observations are mainly limited to $\mathrm{Ca}$ II $\mathrm{H}$ and K (e.g., Jensen \& Orrall 1963; Beckers 1968; Liu 1974; Teplitskaia \& Firstova 1976; Cram \& Dame 1983; Rutten \& Uitenbroek 1991; Lites et al. 1999; Rezaei et al. 2007; Beck et al. 2008b; Teplitskaya et al. 2009), H $\alpha$ (e.g., Bray 1973; Schmieder et al. 1984; Tsiropoula et al. 1993; Balasubramaniam et al. 2004; López Ariste et al. 2005; Sánchez-Andrade Nuño et al. 2008; Cauzzi et al. 2009; Bostanc1 2011), the Ca II IR triplet (e.g., López Ariste et al. 2001; Socas-Navarro 2005; Pietarila et al. 2007b; Cauzzi et al. 2008; Vecchio et al. 2009), and He I

^ The animation is available in electronic form at http: //WwW . aanda.org at $1083 \mathrm{~nm}$ (Lites 1986; Rüedi et al. 1995; Socas-Navarro \& Elmore 2005; Sánchez-Andrade Nuño et al. 2007; Kuckein et al. 2009; Felipe et al. 2010) by the absorption in the Earth's atmosphere, whereas from space $\mathrm{Mg}$ II $\mathrm{h}$ and $\mathrm{k}, \mathrm{L} \alpha$, and other lines in the extreme UV are also accessible (e.g., Kneer et al. 1981; Bonnet 1981; Fontenla et al. 1988; Carlsson et al. 1997; Curdt et al. 2010) ${ }^{1}$.

Even though the Ca II $\mathrm{H}$ and $\mathrm{K}$ lines provide a tremendous amount of information on all of the lower solar atmosphere, it turned out to be rather difficult to extract this information from observed spectra (Linsky \& Avrett 1970). Part of the spectral lines forms in an atmospheric regime where the gas density is so low that the assumption of instantaneous local thermal equilibrium (LTE) is no longer valid, because collisions are so scarce that the energy is not necessarily distributed evenly over all possible degrees of freedom. A consistent treatment of line formation in non-LTE (NLTE) is, however, at the limits of both the theoretical description and the commonly available computational resources (Solanki et al. 1991; Carlsson \& Stein 2002; Leenaarts et al. 2009; Martínez-Sykora et al. 2012). After a few

\footnotetext{
1 The references here and before are only intended to roughly cover every decade and research group/instrument.
} 
attempts for a direct analysis of Ca II spectra in the quiet Sun (QS) (Liu \& Skumanich 1974) or active regions (e.g., Teplitskaja $\&$ Efendieva 1975) in the $70 \mathrm{~s}$ and $80 \mathrm{~s}$, the topic has not received much attention in recent years. Apart from the inversion code for Ca II K spectra described in Rouppe van der Voort (2002) that is based on Shine \& Linsky (1974a) and the manual inversion method for the line wings of $\mathrm{Ca}$ II $\mathrm{H}$ and $\mathrm{K}$ used in Sheminova et al. (2005) and Sheminova (2012), only the group led by Prof. Teplitskaya seems to have continued with the development of analysis and/or inversion tools for $\mathrm{Ca}$ II $\mathrm{H}$ and $\mathrm{K}$ (Grigoryeva et al. 1991, 2000, 2009; Teplitskaya \& Grigoryeva 2009).

Many other studies dealt with Ca II $\mathrm{H}$ and $\mathrm{K}$ only in the forward modelling direction, i.e., creating spectra from a model atmosphere to match some average characteristic profile (e.g., Linsky \& Avrett 1970; Shine \& Linsky 1974b; Suemoto 1977; Kneer \& Mattig 1978; Lites \& Skumanich 1982; Solanki et al. 1991), but not with the intention of analysing individual spectra in a two-dimensional (2D) field of view (FOV). The formation of Ca II H spectra was addressed in numerical NLTE simulations (e.g., Rammacher \& Ulmschneider 1992; Rammacher \& Cuntz 2005; Carlsson \& Stein 1997), but again within the forward modelling approach, i.e., the synthesis of Ca II spectra from the output of a numerical model (see also Uitenbroek 2011). While this provides consistent NLTE spectra, it does not allow one to analyse observed spectra directly.

For photospheric spectra, several inversion codes exist that allow one to retrieve physical parameters of the solar atmosphere from observed spectra on an optical depth scale (e.g., Ruiz Cobo \& del Toro Iniesta 1992; Socas-Navarro et al. 2001). With (a few) additional assumptions, the inversion results can then also be converted to an absolute geometrical height scale (e.g., Sánchez Almeida \& Lites 2000; Puschmann et al. 2005, 2010a; Beck 2011). This geometrical height scale is essential for determining many quantities derived from the magnetic field topology, such as the electric current density or the helicity in sunspots (Puschmann et al. 2010b; Ruiz Cobo \& Puschmann 2012). For observations of chromospheric spectral lines, similar inversion codes are only partially available (Socas-Navarro et al. 1998; Tziotziou 2007). For a possible application to Ca II H spectra, the only new development is the NLTE inversion code NICOLE based on Socas-Navarro et al. (2000) that was used in, e.g., Socas-Navarro et al. (2006), Pietarila et al. (2007a) and de la Cruz Rodríguez et al. (2012). However, it still needs to be tested whether it can be successfully applied to Ca II H spectra.

For the analysis of Ca II H spectra, one therefore has to rely on methods that are (hopefully) insensitive to NLTE effects, using observed quantities that are as direct as possible to derive solar atmospheric properties (e.g., Beck \& Rammacher 2010). In the first two papers of this series (Beck et al. 2009, 2012, BE09 and BE12 in the following), we investigated the statistics of velocity and intensity oscillations in Ca II spectra, using only $\mathrm{Ca}$ II $\mathrm{H}$ for the first study, and then added Ca II IR at $854 \mathrm{~nm}$ for the latter thanks to new observations covering both lines simultaneously. The conversion from observed intensities or velocities to energy in these two studies had to rely on some assumptions, such as the LTE condition, that have an impact on the final result. A consistent NLTE analysis would be strongly preferred, but lacking a suitable method for the automatic treatment of the observations with several 10000 spectra each, we first developed and applied an LTE inversion strategy for Ca II spectra based on the SIR code (Ruiz Cobo \& del Toro Iniesta 1992) as a preliminary step to a possible full NLTE treatment in the future. The LTE assumption does not fail instantly in the solar atmosphere, but results derived assuming LTE will deviate more and more from the "reality" the closer one gets towards the very line core of $\mathrm{Ca}$ II $\mathrm{H}$ that forms highest above the continuum. The LTE inversion can also be helpful for an NLTE analysis because it both outlines a suitable approach for the inversion procedure and provides a reasonable initial model for the NLTE fit that presumably will speed up its convergence.

The observations used are outlined briefly in Sect. 2, whereas Sect. 3 describes the stray-light correction. The LTE inversion approach is described in Sect. 4. Section 5 shows the result of the application of the code to the spectra. The results are discussed in Sect. 6. Section 7 provides our conclusions. Appendix A explains the animation of the inversion results that accompanies this paper.

\section{Observations}

For the present study, we used the Ca II H spectra of the QS observations on disc centre labelled Nos. 1 and 2 in BE12. The first observation is a large-area scan taken on 24 July 2006 that was used in BE09 before and is described in detail there. It consists of simultaneous spectroscopy of $\mathrm{Ca}$ II $\mathrm{H}$ and spectropolarimetry at $630 \mathrm{~nm}$ obtained with the POlarimetric LIttrow Spectrograph (POLIS, Beck et al. 2005) at the German Vacuum Tower Telescope (VTT, Schröter et al. 1985) in Izaña, Tenerife, Spain. The integration time per scan step was $6.6 \mathrm{~s}$. The image of the solar surface was scanned in 150 steps of 0.5 step width, covering a total FOV of about $75^{\prime \prime} \times 70^{\prime \prime}$. The slit width was 0.5 , and the spatial sampling along the slit was 0.3 . The second observation is a time series taken with POLIS on the same day that is described in detail in Beck et al. (2008b, BE08). The integration time was $3.3 \mathrm{~s}$ per scan step and the cadence of repeated co-spatial spectra is about $21 \mathrm{~s}$.

For comparison with the results for these QS data on disc centre we also applied the inversion code to an observation of an active region (AR) at a heliocentric angle of about $50^{\circ}$. These data were taken on 8 December 2007 with a combination of POLIS and the Tenerife Infrared Polarimeter (TIP, Martínez Pillet et al. 1999; Collados et al. 2007). The data consist of three scans of 200 steps with a step width of $0{ }^{\prime} 5$, covering a large part of the active region NOAA 10978. The integration time was $3.3 \mathrm{~s}$ per scan step. The rest of instrumental characteristics for POLIS were as above. Overview maps of these observations can be found in Fig. 16 in Beck \& Rammacher (2010). One of the AR maps is described in detail in Bethge et al. (2012). All data were taken with a real-time seeing correction by the Kiepenheuer-Institute adaptive optics system (KAOS, von der Lühe et al. 2003). The spatial resolution of the observations, being slit-spectrograph data, cannot be improved post facto by image reconstruction techniques (e.g., Puschmann \& Beck 2011) apart from a possible correction for the instrumental point spread function (Beck et al. 2011, BE11 in the following).

\section{Stray-light correction}

The Ca II H channel of POLIS has two major contributions from stray light (BE11): a spectrally undispersed ("parasitic") part, $\beta$, caused by scattering inside of the spectrograph itself, and spectrally resolved stray light, $\alpha$, caused by optics inside and in front of POLIS. For the current investigation, we used a simplified version of the stray-light correction described in BE11. The observed spectra after subtracting the dark current and flat fielding, $I_{\text {raw }}$, were first corrected for the prefilter transmission curve 
C. Beck et al.: The energy of waves: LTE inversion of Ca II H spectra

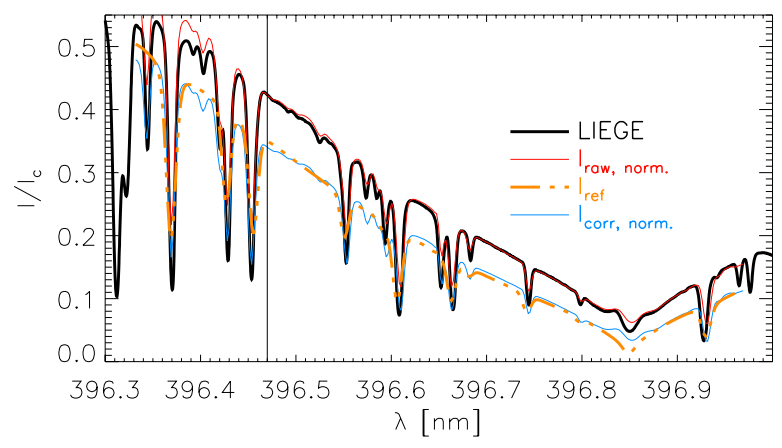

Fig. 1. Intensity normalization and stray-light correction of the average Ca II H profile. Thick black: LIEGE atlas profile. Thin red: normalized average observed profile $I_{\text {raw,norm. }}$. Thick orange dash-dotted: reference profile of the modified HSRA model. Thin blue: stray-light corrected average observed profile $I_{\text {corr.norm. }}$. The black vertical line denotes the wavelength of intensity normalization.

and normalized on average to the Liege spectral atlas (Delbouille et al. 1973) in the line wing near $396.5 \mathrm{~nm}$ (Fig. 1). The normalized spectra $I_{\text {raw,norm. }}$ were then corrected for stray light by

$I_{\text {corr.,norm. }}=\left(I_{\text {raw,norm. }}-0.025\right) \times 0.85$.

This corresponds to assuming a parasitic stray-light level of $\beta=$ $2.5 \%$ of $I_{\mathrm{c}}$ and a spectrally resolved stray-light level of $\alpha=15 \%$. The former value is identical to the one derived in BE11 $(5 \%$ of the intensity at $396.4 \mathrm{~nm} \sim 2.5 \%$ of $I_{\mathrm{c}}$ ), whereas $\alpha$ is slightly lower.

The values of $\alpha$ and $\beta$ used here were determined to match the average observed profile and a reference profile related to the inversion of the spectra. The reference profile (Fig. 1) corresponds to the LTE synthesis of the Ca II H spectrum for a model atmosphere without a chromospheric temperature rise constructed from a combination of the Harvard Smithsonian reference atmosphere (HSRA, Gingerich et al. 1971) and the Holweger-Mueller model (HOLMUL, Holweger $\&$ Müller 1974). The exact shape of the reference profile in the line core, i.e., from $396.83 \mathrm{~nm}$ to $396.88 \mathrm{~nm}$, where the emission peaks and the central absorption core are located, is uncritical because only the line wings are matched by the straylight correction, not the line core. Therefore the choice of which temperature stratification to use for creating the reference profile (HOLMUL, HSRA, VAL, FAL; Vernazza et al. 1981; Fontenla et al. 2006) is not important because all these theoretical atmosphere models only differ significantly in chromospheric layers, hence in the shape of the line core, whereas the line wing is similar for spectra synthesized from any of the models.

\section{LTE inversion}

Initial attempts to fit the Ca spectra with the SIR code (BE08, Appendix B) showed that the code tends to ignore the line-core region in the fit. This is caused by the equal weight used for every wavelength point, which is perfectly suitable for photospheric lines, but not for chromospheric ones. The line core and the neighbouring wavelength points $( \pm 0.05 \mathrm{~nm} \equiv \pm 25$ pixels of $1.92 \mathrm{pm}$ in the POLIS spectra) contain all the information about the "upper" part of the atmosphere $(\log \tau<-2)$, whereas the remaining $\sim 270$ wavelength points contain (redundant) information about the lower atmosphere. The intensity near the line core is the lowest of the full spectrum, and therefore any mismatches between synthetic and observed spectra stay small and have no strong effect on the least-square value to be minimized in the fit.
Table 1. Parameter ranges used in generating the archive.

\begin{tabular}{lccc}
\hline \hline$T_{\text {diff }}[\mathrm{K}]$ & $\left.T_{\text {ampl }}\right|_{\tau=1}[\mathrm{~K}]$ & $\sigma[\log \tau]$ & $P[\log \tau]$ \\
\hline$-300-+300(50)$ & $10-100(10)$ & $0.3-3.1(0.1)$ & $-6-+1.4(0.1)$ \\
\hline
\end{tabular}

Notes. The values in parentheses give the step width.

A second problem is that the temperature stratifications required to reproduce the observed spectra may have very complicated shapes such that an iterative modification of a given simple initial model may never result in the necessary complex shape, regardless of the number of nodes used for modifying the temperature stratification during the fit. To overcome this limitation in the inversion of the Ca II H spectra, we used a comparison of the observed spectra to a pre-calculated archive of $\mathrm{Ca}$ II H spectra as first step, giving an increased weight to the line core at this step. Similar archives have been used in inversion approaches of other chromospheric lines before, mainly for $\mathrm{H} \alpha$ (Molowny-Horas et al. 1999; Tziotziou et al. 2001; Schmieder et al. 2003; Berlicki et al. 2005).

\subsection{Creation of an archive of LTE spectra}

The base of the archive is the modified version of the HSRA that emulates an atmosphere in radiative equilibrium. The archive profiles were then generated by adding temperature perturbations of varying shapes and amplitudes to the modified HSRA model and synthesizing the resulting spectra with the SIR code. SIR uses complete frequency redistribution and assumes LTE conditions. We only modified the temperature and kept all other parameters as given in the modified HSRA model.

One example of the temperature perturbations applied in creating the archive had a Gaussian shape, with different central positions $P$ in $\log \tau$, widths $\sigma$, and amplitudes $T_{\text {ampl }}($ at $\log \tau=0$ ). The ranges for the three parameters are given in Table 1 . The temperature amplitude for the Gaussian was scaled up with its location in optical depth by the square root of the electron pressure, normalized to its value at $\log \tau=0$ (cf. the top panel of Fig. 2). The Gaussian perturbation was then moved across all 75 grid points in optical depth from $\log \tau=1.4$ to -6 . An example of the resulting temperature stratifications for one run of the Gaussian through optical depth is given in the middle panel of Fig. 2. The lower panel shows the resulting synthetic spectra. This setup was chosen because spectra from a single run in optical depth roughly reproduce the temporal evolution of observed spectra during the occurrence of a bright grain (see the right panel of Fig. 4 later on). Because of the scaling of the temperature amplitude with optical depth, an initial amplitude $T_{0}$ at $\log \tau=0$ of, e.g., $100 \mathrm{~K}$ converts to a perturbation with an amplitude of about $4000 \mathrm{~K}$ when the Gaussian is located at $\log \tau \sim-4$. The archive thus automatically covers a wide range of possible temperature values. We additionally globally added (subtracted) up to $\pm 300 \mathrm{~K}$ to (from) all depth points ( $T_{\text {diff }}$, first column of Table 1) when running the Gaussian perturbation through optical depth. Additional synthetic spectra were created by adding (subtracting) straight lines of variable slope to (from) the modified HSRA model instead of adding a Gaussian perturbation.

Line-of-sight (LOS) velocities were not included in generating the archive profiles because this would have increased the size of the archive beyond usefulness. Velocities are instead taken into account in an empirical way in a later step. The spectral resolution of the Ca II $\mathrm{H}$ spectra from POLIS corresponds to a velocity dispersion of about $1.5 \mathrm{~km} \mathrm{~s}^{-1}$ per pixel. Together with 

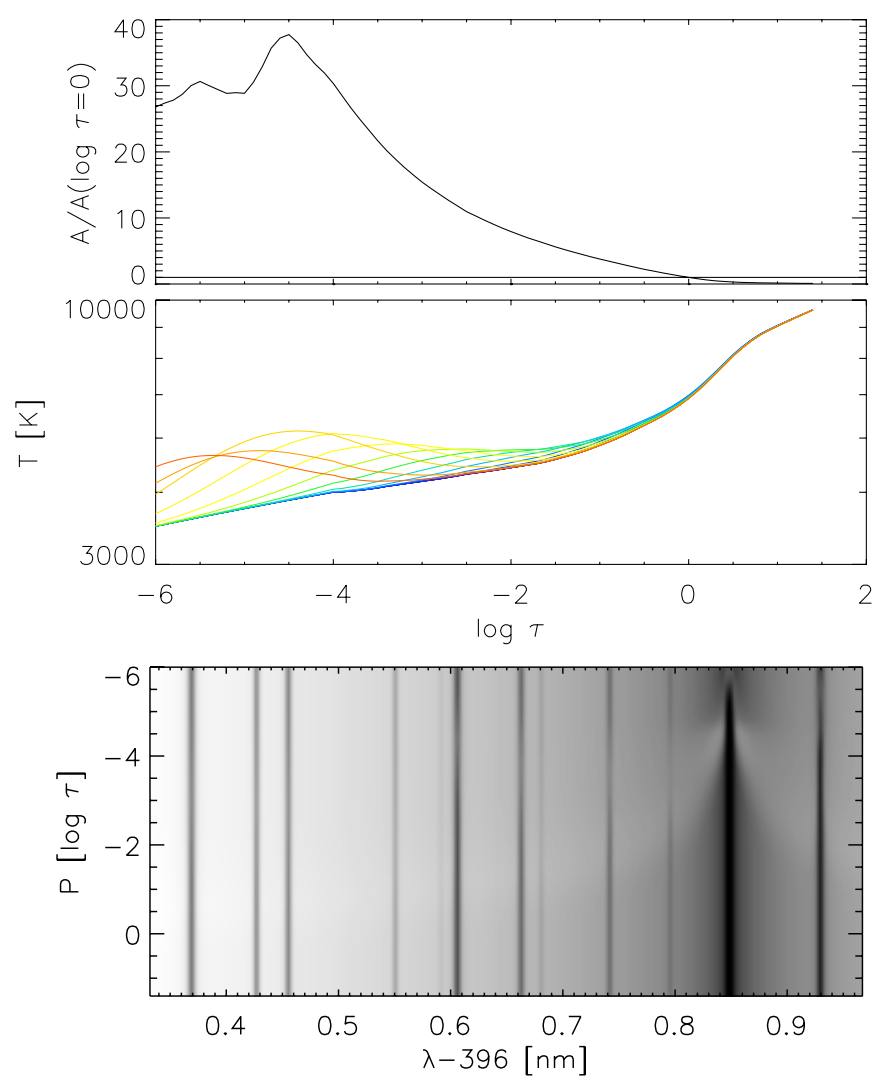

Fig. 2. Top: scaling law for the temperature amplitude with optical depth. Middle: example of temperature stratifications with the Gaussian perturbation centred subsequently at decreasing optical depth. Bottom: the 75 synthesized spectra corresponding to the temperature stratifications above for the Gaussian perturbation centred subsequently at the location $P$ from +1.4 (lowermost spectrum) to -6 (topmost spectrum) in $\log \tau$.

the intrinsic (thermal) width of spectral features, the weak velocity response allowed us to still use the pre-calculated archive spectra as the first step in fixing the temperature stratification because the Doppler shifts (in pixel) stay comparably small. The macroturbulent velocity in the atmosphere was set to a fixed value of $1 \mathrm{~km} \mathrm{~s}^{-1}$ in the generation of the archive spectra, which yielded line widths for the photospheric line blends that match the observed line widths.

With the Gaussian perturbations defined by the free parameters $\left(T_{\text {diff }}, P, \sigma, T_{\text {ampl }}\right)$, and the other additional modifications of the temperature stratification, in total more than 300000 Ca II H spectra were synthesized with SIR. All line blends of $\mathrm{Ca}$ II $\mathrm{H}$ with known transition parameters were taken into account (cf. Table 1 in BE09). We also always synthesized the corresponding spectra at $630 \mathrm{~nm}$ from the atmospheric models because POLIS provides both wavelength ranges simultaneously. The creation of the archive by a single (background) process takes about one week.

\subsection{Best-fit spectrum from LTE archive}

To find the archive profile $A(\lambda, i)$ ( $i$ counts the number inside the archive) that matches a given observed profile $I(\lambda)$ best, the least-square deviation between the observed profile and all archive profiles was calculated by

$\chi_{i}^{2}=\sum_{j} w\left(\lambda_{j}\right) \cdot\left(I\left(\lambda_{j}\right)-A\left(\lambda_{j}, i\right)\right)^{2}$.
The weights $w\left(\lambda_{j}\right)$ were set to the squared inverse of an average QS profile, $w=1 / I_{\mathrm{av}}^{2}$, with a subsequently doubled weight for the region around the line core itself from about $396.81 \mathrm{~nm}$ to $396.89 \mathrm{~nm}$. This strongly enhances the influence of the line-core intensities relative to the wing (ratio of about 4:1). All (strong) line blends were masked out by setting the weights to zero at these wavelengths. The $630 \mathrm{~nm}$ spectra were not included in the $\chi^{2}$ for finding the best-matching archive profile because the fit to the $\mathrm{Ca}$ line core is the main driver for this step.

\subsection{Iterative improvement of temperature stratification}

The best match of observed and archive profiles is determined with a strong weight for the line core. In the line wing, the observed and best-fit archive profiles usually deviate because the archive only contains a limited number of all of the possible combinations of relative wing and core intensities. To improve the fit in the line wing, we used an iterative modification of the temperature stratification based on the intensity differences between observed and synthetic profiles at some wavelengths. The temperature values at the corresponding layers in $\log \tau$ that contribute to the intensity at these wavelengths are then modified according to the modulus and sign of the intensity differences.

We selected a series of wavelength windows without photospheric blends and the blue part of the line core up to the line centre (Fig. 3). For these wavelengths $\lambda$, we calculated the corresponding layers in $\log \tau$ using the intensity response function of $\mathrm{Ca}$ II $\mathrm{H}$ in the modified HSRA model (cf. Rezaei et al. 2008, BE09). The intensity response function matches well the formation heights derived from phase differences of propagating waves (BE08, BE09). We then implemented the relation between $\lambda$ and corresponding $\tau$ into the code (provided by an external file). It turned out that the hardwired response function works fine for all spectra on the disc centre, but for the AR observations at a heliocentric angle of $50^{\circ}$, the relation between $\lambda$ and $\tau$ was already slightly off, leading to a misfit in the outermost wing/lowermost atmosphere layers. It seems therefore to be recommended to at least re-calculate the average $\lambda-\tau$ relation for an application to off-centre observations.

During the iterative improvement of the complete temperature stratification the simultaneous $630 \mathrm{~nm}$ spectra were now included by adding one more point to the wavelength windows used. This point was located in the continuum of the $630 \mathrm{~nm}$ channel, corresponding to $\log \tau \sim 0.2$ in the intensity response. The lowest forming wavelengths in the $\mathrm{Ca}$ wing in the usual setup of POLIS form at about $\log \tau \sim-0.2$ (Fig. 3). The use of the $630 \mathrm{~nm}$ continuum intensity was, however, implemented only optionally and can be skipped when no $630 \mathrm{~nm}$ data are available.

With the relation between $\lambda$ and $\tau$ given, one can then modify the temperature stratification according to the intensity differences between observed and best-fit spectrum, $\Delta I(\lambda)$, at the wavelengths considered. The intensity difference is first converted to a temperature difference using the intensity response function. We then fit a fourth-order polynomial to the required temperature changes in $\log \tau$ to obtain a smooth curve for all optical depth values. The slope of the curve is extrapolated towards the layers with $\log \tau>0.2$ where no measurements are available. The change in the temperature stratification is then added to the previous stratification, and the new spectrum is synthesized. The middle panels of Fig. 3 show some examples of the variations during the iteration of the temperature stratification. The method converges usually on two or three iterations. The code uses no 

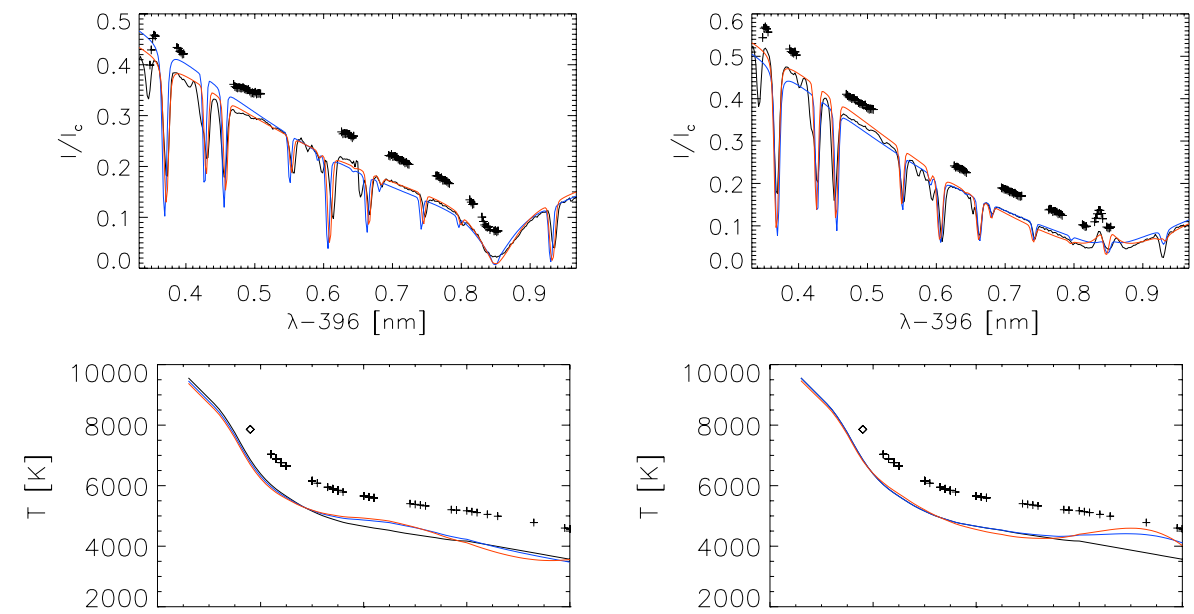

Fig. 3. Iterative improvement of the best-fit archive spectrum for two example spectra without (left column) and with emission in the line core (right column). Top row: observed spectrum (black line), best-fit archive spectrum (blue), and final best-fit spectrum (red). The black crosses denote the wavelengths and
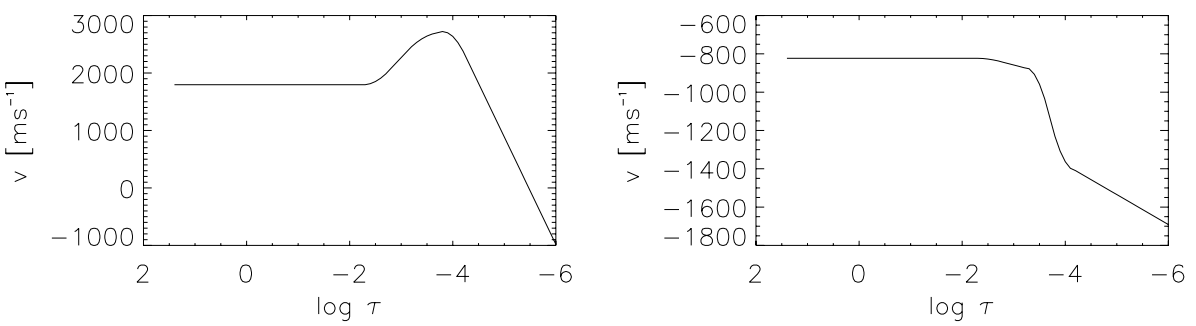
intensities used for the improvement of the fit. 2nd row: temperature stratifications (blue: archive best-fit, red: final best-fit, black: modified HSRA model). The black crosses denote the optical depths corresponding to the wavelengths marked above. The diamond at $\log \tau=$ 0.2 denotes the $630 \mathrm{~nm}$ continuum intensity contribution. Bottom row: stratification of the LOS velocity.

special convergence criterion, but the number of iterations to be done has to be provided by the user.

In the iteration, no special weight is given to the line core because this step is mainly intended to improve the fit in the line wing. As a result, the $\chi^{2}$ value using the weighting with an enhanced contribution of the line core (cf. the previous section) can actually worsen. Even if this happens, the iterated temperature stratification is returned because otherwise it is not possible to obtain a good fit to the line wing. To identify such cases, i.e., a degradation of the fit to the $\mathrm{Ca}$ line core in the iteration process, the inversion routine returns an additional binary value of 0 or 1 that indicates a decrease or increase in the weighted $\chi^{2}$ (cf. Sect. 6.1 later on).

The LTE condition in the spectral synthesis of the profiles requires the temperature stratifications to have a negative slope in the uppermost layers for creating double reversals in the line core (cf. the middle right panel of Fig. 3). This usually affects the last three to five grid points of the optical depth scale $(\log \tau=-5.5$ to -6$)$ at the upper end of the optical depth scale. The profiles created by the addition of the Gaussian perturbation to the modified HSRA automatically have such a negative slope whenever the centre of the Gaussian is not located exactly at $\log \tau=-6$.

\subsection{Inclusion of LOS velocities}

In the iterative improvement of the temperature stratification, the LOS velocities are finally included in an empirical way. We use three of the line blends of Ca II H (CrI at $396.37 \mathrm{~nm}$, Fe I at $396.61 \mathrm{~nm}, \mathrm{Fe} \mathrm{I}$ at $396.93 \mathrm{~nm}$ ) and the $\mathrm{Ca}$ line core itself. The line blends in the wing correspond to three different formation heights (cf. BE09). We use the observed LOS line-core velocities of the four lines to construct a velocity stratification by attributing the observed velocities to the corresponding $\log \tau$ layers of $-2.4,-3.4,-3.9$, and -6 , and then linearly interpolate between the velocity values. To prevent step functions, the resulting velocity curve with optical depth is smoothed. The bottom panels of Fig. 3 show the resulting velocity stratification for the observed spectra in the top panels. The velocity is not modified to obtain a better fit, but only serves to yield a better match of synthetic and observed spectra using the observed velocity values. For the line blends, the velocity is determined well, whereas for the $\mathrm{Ca}$ line core the location of the line minimum in some cases might not reflect a true velocity at all.

For the inversions of the AR maps, we did not include LOS velocities because the line core of $\mathrm{Ca}$ II $\mathrm{H}$ often has a complex shape there with a single (or several) intensity reversal that does not easily allow one to ascribe any velocity to it. Because of the weak response of the spectra to LOS velocities, this should have had a negligible effect on the retrieved temperatures.

It takes about eight seconds to obtain a fit to a single observed profile on a common desktop computer, hence about three days for all observed spectra in one typical observation of 30000 profiles.

\section{Results}

\subsection{Example spectra in the quiet Sun}

For a visual inspection of the quality of the fits, we show a series of spectra in the following Figs. 4 to 8 . The left-hand panel of Fig. 4 shows the observed and best-fit Ca spectra (first and second columns) for three locations along the slit in the time series in QS. The third column shows the corresponding temperature stratifications. The fourth column shows the simultaneous and co-spatial Stokes $V$ spectra at $630 \mathrm{~nm}$ to demonstrate the presence (or absence) of photospheric magnetic fields. The upper row corresponds to a location in or near the photospheric network (see also Beck et al. 2008a). An animation of the spectra along the slit and the corresponding inversion results for all time steps is available in the online material (see Appendix A).

Comparing observed and best-fit spectra in Figs. 4 to 6 , we find that the inversion approach is able to deal well with the spectral range from the wing up to about $396.8 \mathrm{~nm}$, fully reproducing the intensity patterns and the Doppler shifts of the line blends. 

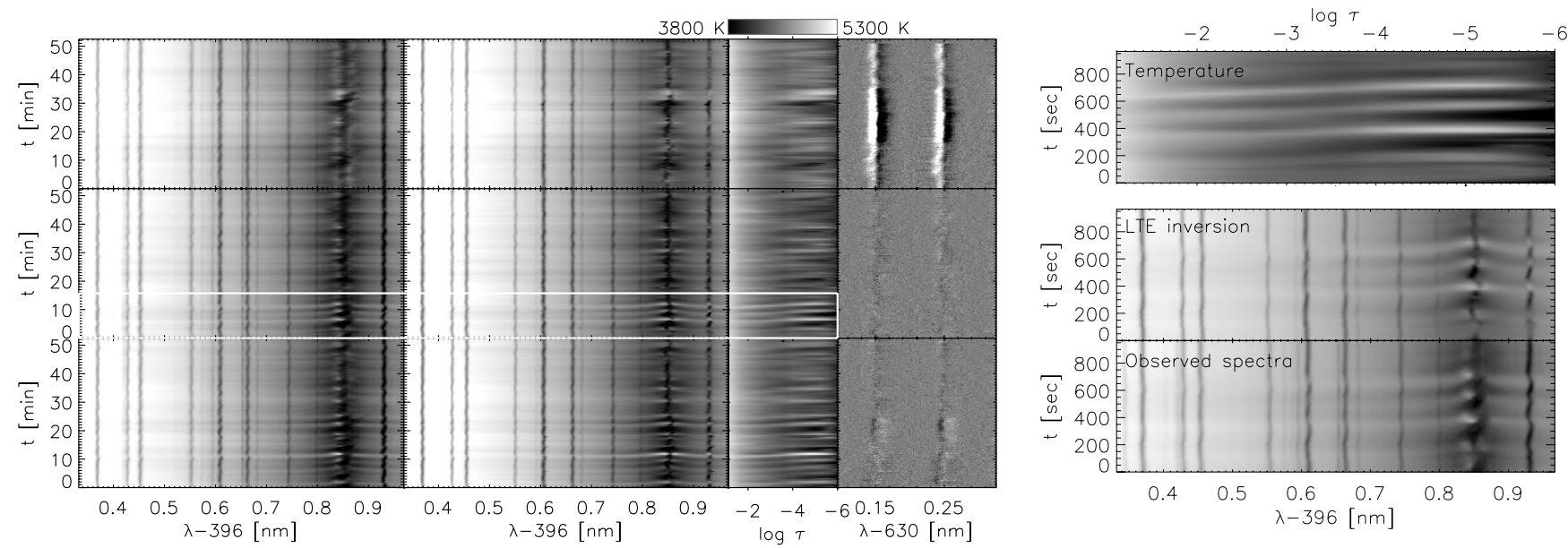

Fig. 4. Comparison of observed spectra and inversion results in the time series. Left panel, top to bottom: spectra from three different locations along the slit versus (vs.) time. The upper row corresponds to a network location. First column: observed spectra. Second column: best-fit spectra. The third column shows the temperature stratifications vs. $\log \tau$, the fourth column the simultaneous Stokes $V$ spectra of the $630 \mathrm{~nm}$ channel. The white rectangle in the second row marks the section shown magnified on the right-hand side. A train of three consecutive brightenings passes from the line wing towards the line core between $t=300 \mathrm{~s}$ and $t=800 \mathrm{~s}$.
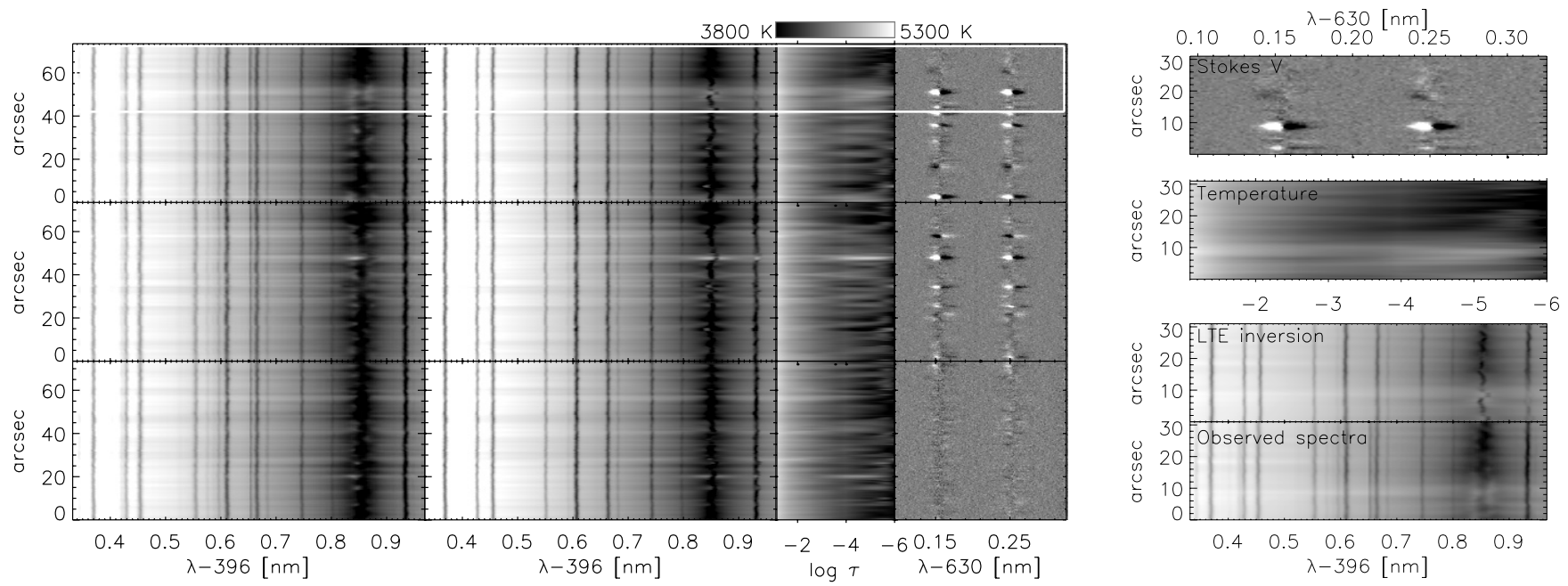

Fig. 5. Same as Fig. 4 for the large-area scan. This time the cuts are along a spatial axis and do not represent a temporal evolution. The white rectangle in the top row marks the section shown magnified on the right-hand side.

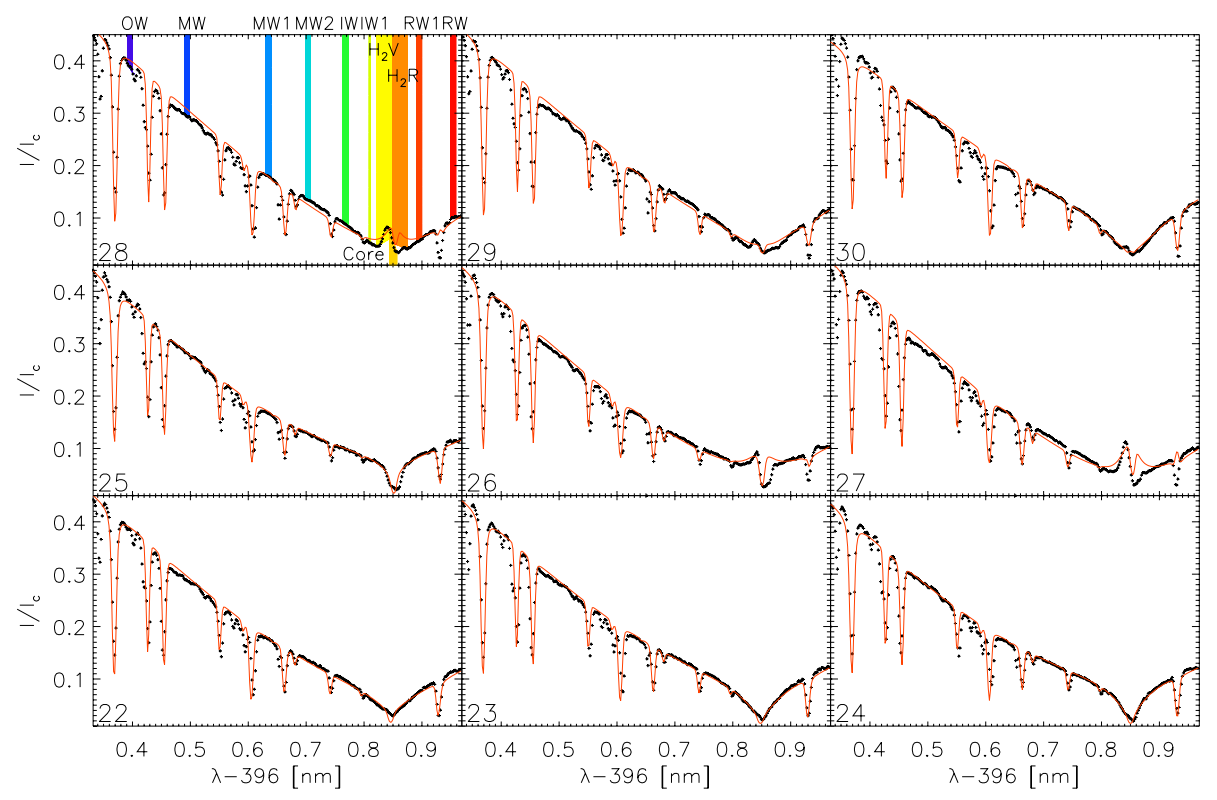

Fig. 6. Example spectra during the passage of a (shock) wave. Observations are given by black pluses and the best-fit spectra by red lines. The temporal cadence between subsequent spectra is $21 \mathrm{~s}$. Time increases from left to right in each row and from bottom to top between rows. The coloured bars in the upper left panel denote the wavelength bands used later on. 


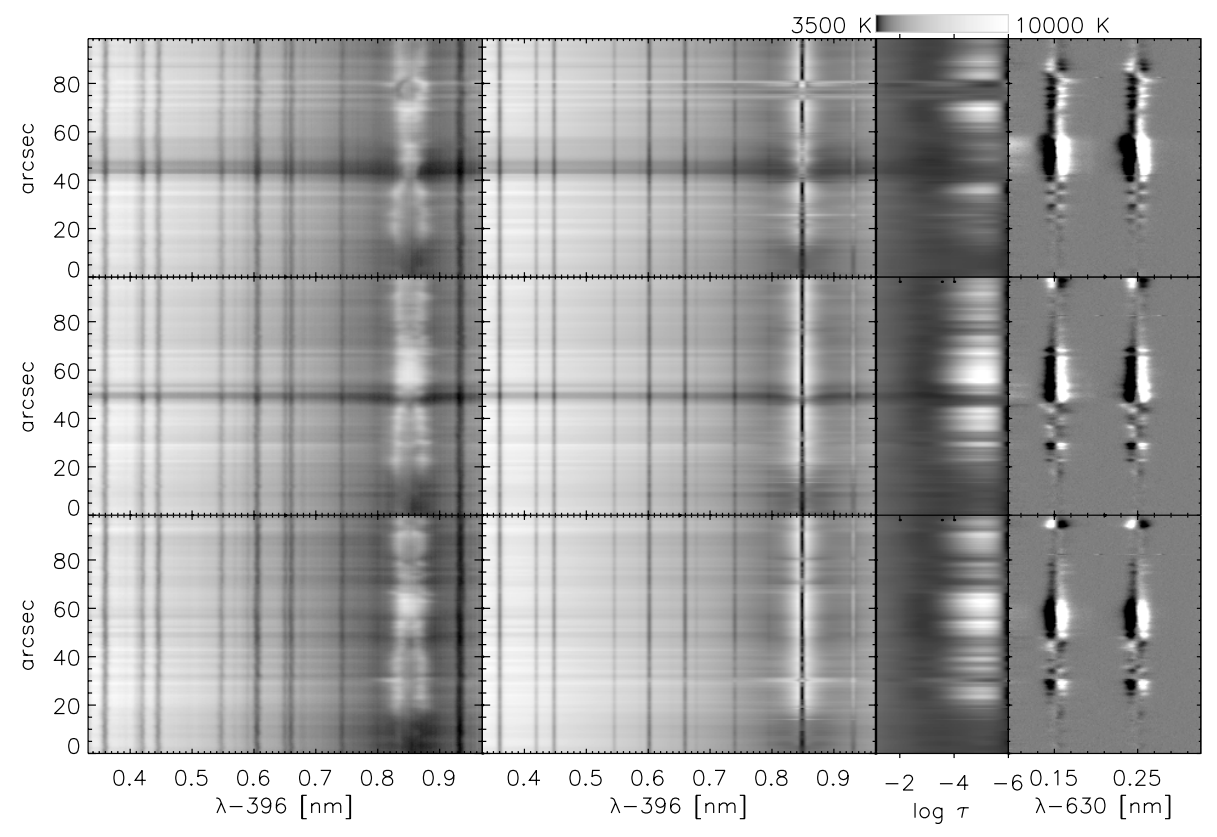

Fig. 7. Same as Fig. 5 for three cuts through the active region. No LOS velocities were considered in this fit. The temperature is displayed on a logarithmic scale in a range from $3500 \mathrm{~K}$ to $10000 \mathrm{~K}$ this time.
In the Ca core, the red emission peak is in general always reproduced worse than the blue one. This is caused by the inability to reproduce the line-core region without including NLTE effects and more complex velocity fields than used in the inversion (cf. Carlsson \& Stein 1997).

The core of the spectral line of Fe I at $396.93 \mathrm{~nm}$ reverts to emission in the best-fit spectra at several locations, contrary to the observations (e.g., at $t=400,600,750$ secs in the right panel of Fig. 4). The LTE assumption thus already fails for the core of this line in some cases. The temporal evolution, e.g., wave trains propagating from the line wing towards the core, and all variations in the line wing are captured well by the inversion. The behaviour of the observed spectra during the passage of three consecutive wave fronts (right panel of Fig. 4) is well matched except for the asymmetry of the red and blue emission peaks. The corresponding temperature stratifications show both the upwards propagation of the wave fronts and the increase in their amplitude in the upper atmosphere.

The differences between magnetic and field-free locations can be seen better in Fig. 5, which shows spatial cuts through the large-area map. Whereas the (temporary) enhancements typical of propagating waves often only appear in the upper layers $(\log \tau<-4)$ with a reduction of temperature between about $\log \tau=-2$ to -4 (cf. the top panel at the right side of Fig. 4), the temperature at most locations with a significant polarisation signal, i.e., magnetic fields, is usually increased over the complete optical depth range (third column of the left panel of Fig. 5). The magnification on the right-hand side of Fig. 5 shows that in some cases regions with reduced intensity in the line core can extend spatially connected over $5-10^{\prime \prime}$, reflected by the corresponding reduction of temperature at $y=20-30^{\prime \prime}$, in contrast to the magnetic location around $y=9^{\prime \prime}$.

The accuracy in the reproduction of the observed profiles in the QS is visualized in more detail in Fig. 6, which shows the evolution of individual spectra during about three minutes in the time series. In that time span, one wave front passes through the $\mathrm{Ca}$ line formation range, culminating in strong emission of the $\mathrm{H}_{2 \mathrm{~V}}$ peak when reaching the line core. The intensity near the line core rises gradually (profiles Nos. 23 and 24), yielding a profile with an elevated line wing close to the line core and a narrow absorption core without emission peaks in
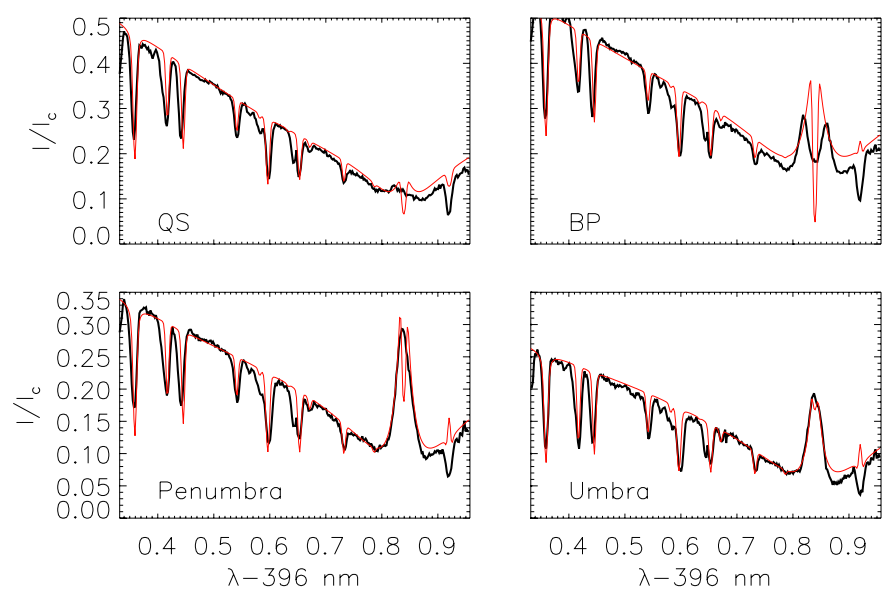

Fig. 8. Individual spectra from the active region map. Clockwise, starting left top: quiet Sun, bright point in QS, umbra, penumbra. Black: observed spectrum. Red: best-fit spectrum.

profile No. 25 (leftmost middle panel of Fig. 6), which gives way to one with a strong $\mathrm{H}_{2 \mathrm{~V}}$ emission peak 20-40 s later at profile No. 27 (rightmost middle panel of Fig. 6). The emission in the line core subsides again after the passage of the shock front (profiles Nos. 28 to 30), whereas at the end in the line wing the next wave front is already indicated (compare profiles Nos. 24 and 30). The individual profiles clearly demonstrate that the $\mathrm{H}_{2 \mathrm{R}}$ emission peak is not reproduced by the fit, but the amplitude of $\mathrm{H}_{2 \mathrm{~V}}$ is matched well, e.g., in profile Nos. 26 to 29 .

\subsection{Example spectra in active region off disc centre}

Figure 7 shows observed and best-fit spectra for three spatial cuts through one of the three active region maps in the same layout as in the left-hand panel of Fig. 4. The best-fit spectra differ more from the observed spectra than for the QS data on disc centre. No LOS velocities were included in the fit, because for the line core of $\mathrm{Ca}$ II $\mathrm{H}$ it is difficult to determine any suitable value directly from the spectra. The profiles in the umbra show only a single emission peak (where a fit of a Gaussian could be used), those in the penumbra usually double reversals (where 
the location of the central absorption could be used), but also mixtures of these two types or profiles with a plateau of emission appear. A determination of LOS velocities to be attributed to the $\mathrm{Ca}$ line core would thus require a (predetermined) choice of the method to derive the velocity because it is impossible to use a unique method over the complete FOV. However, because of the small impact of LOS velocities up to the sound speed on the spectra, neglecting the post facto inclusion of velocities should not have influenced the retrieved temperature stratifications.

Nevertheless, a clear deviation between best-fit and observed profiles is seen in the actual line core of Ca II H. All best-fit profiles outside the umbra are characterized by a central absorption core that is seen in neither the observed AR spectra nor the QS data. This fact is more visible in the individual spectra of Fig. 8 that correspond to positions in the umbra, penumbra, a bright point (BP) in a plage area, and a "QS" location in the AR. Except for the umbral profile, all best-fit profiles have a dark central absorption core. This absorption core presumably results from the requirement to have a decrease in the temperature in the uppermost layers to obtain "reasonable" spectra (imposed by the LTE assumption, cf. also Rezaei et al. 2008). It shows up as much more prominent for the AR LTE spectra because there a relatively strong chromospheric temperature rise is required to produce the strong emission core at first, on whose top the (small) absorption core is then superposed. In the QS spectra, the strong emission core is absent. Another difference between Figs. 5 and 7 is the temperature range in the AR required for displaying the temperature stratifications in the third column of Fig. 7. The variations in temperature are so much larger than in QS that the display range had to be significantly increased, even while the display now is on a logarithmic scale.

The creation of the archive spectra was done in a semiautomatic way without explicitly taking differences between spectra in QS and AR into account. Figure 8 demonstrates that the archive also covered by chance the range of profile shapes that are observed in ARs.

\section{3. $2 D$ maps in the quiet Sun}

The lower panel of Fig. 9 shows 2D spatio-temporal maps of the intensity in selected wavelength bands as a final cross-check of the performance across the complete FOV and the full spectral range (compare to, e.g., Cauzzi et al. 2008, their Figs. 2 and 3). The wavelength bands are described in more detail in Rezaei et al. (2007) and BE08, and are marked in the upper left-hand panel of Fig. 6. They pass from the outer line wing (OW) in the blue through the line core towards the red line wing (RW), avoiding the line blends. The only differences between observed and inverted spectra that can be discerned by eye are in the map of the continuum intensity at $630.4 \mathrm{~nm}$ and in the $H_{2 \mathrm{R}}$ map. For the former, the dynamic range of the observed continuum intensity at $630.4 \mathrm{~nm}$ is not fully matched and the spatial pattern in the inversion spectra is more similar to that in the OW than to the observed continuum intensity itself. This presumably is related to the weak influence of the continuum intensity in the iterative improvement of the fit because it provides only one wavelength, hence one optical depth point to the modification of the temperature stratification (Sect. 4.3). For $\mathrm{H}_{2 \mathrm{R}}$, the observations show a higher intensity than the fit for the network regions at $y=10-20^{\prime \prime}$ and around 50".

The upper panel of Fig. 9 shows temperature maps at several optical-depth values for the inversion of the time series, for a visualization of the relation between observed spectra and the corresponding temperatures. The LTE assumption maps the intensity at some wavelength to the temperature at some optical depth, but because the radiative transfer through the complete depth stratification was considered, the approach exceeds the direct conversion from intensity at one wavelength to temperatures through the Planck function used in, e.g., Cauzzi et al. (2009) or BE12. During the iteration of the best-fit stratification from the LTE archive the code also always modifies the temperature over some range in $\log \tau$ such that the profile after the integration of the radiative transfer equation matches the observed spectrum. This also breaks up the one-to-one correlation between a given wavelength and a single layer in $\log \tau$. By comparing the intensity and temperature maps in Fig. 9, one can identify some features that appear modified in the spectra and the temperature stratification. One case is a large-scale $\subset$-shaped darkening around ( $\left.t=40 \mathrm{~min}, y=25^{\prime \prime}\right)$ that is only prominent in the line-core map of the observations and weakly in $\mathrm{H}_{2 \mathrm{~V}}$ and $\mathrm{H}_{2 \mathrm{R}}$, but can be seen in the temperature maps for all layers above $\log \tau=-4.5$.

Figure 10 shows the intensity maps in the wavelength bands in the large-area scan in QS on disc centre. For the comparison between observed and best-fit spectra in Fig. 10, the match seems to be even better than for the spectra of the time series on disc centre shown in Fig. 9. The increasing spatial extent of the emission near the locations of photospheric network fields in wavelength bands near the line core is clearly seen in both observed and best-fit spectra. Wavelength bands that form lower are also well matched. The largest deviations can be seen in the map of the $\mathrm{H}_{2 \mathrm{R}}$ emission peak (fourth column in the upper two rows), the RW1 map (fifth column in the upper two rows), and the continuum intensity at $630.4 \mathrm{~nm}$. The last two maps show some intensity offset and contrast difference between observations and best-fit spectra.

The temperature maps in the upper panel of Fig. 10 show the extension of the emission around the locations of concentrated photospheric magnetic flux for $\log \tau$ smaller than -4.5 . Isolated temperature enhancements (e.g., at $x, y \sim 65^{\prime \prime}, 50^{\prime \prime}$ ) seem to be more pronounced and localized than the corresponding brightenings in intensity in $\mathrm{H}_{2 \mathrm{~V}}$ or the line-core map. The magnetic locations are not discernible in the temperature map at $\log \tau=-1.5$. The lowest layer displayed at $\log \tau=-0.5$ already clearly forms above the continuum layers and roughly corresponds to the intensity map of MW2.

\section{4. $2 D$ maps in active region}

Several peculiarities stand out in the temperature maps of the AR (Fig. 11). The map shown in the bottom row exhibits two dark filaments for $\log \tau<-4$ at $x \sim 70^{\prime \prime}$ and $y \sim 15^{\prime \prime}$ and $30^{\prime \prime}$, respectively. The lower, larger one has a co-spatial filament in He I spectra at $1083 \mathrm{~nm}$, and was identified with a transient siphon flow by Bethge et al. (2012). Several similar dark filaments that originate or terminate in individual pores can be identified in the scan shown in the middle row. An investigation of the related Doppler shifts will allow one to determine if they are also related to similar siphon flows (cf. Uitenbroek et al. 2006; Beck et al. 2010). The AR map in the top row shows two interesting emission features or, equivalently, locations of strong temperature enhancements. The first is an isolated bright point (BP) at $(x, y) \sim\left(45^{\prime \prime}, 30^{\prime \prime}\right)$. This BP has about the highest temperature of the full FOV at $\log \tau=-3$, and still stands out in the map at $\log \tau=-4$. The Ca spectra at this location are similar to the BP spectra shown in Fig. 10 of Beck et al. (2005). The second large-scale region of temperature enhancements is located just between the large and small sunspot in the top row of Fig. 11 

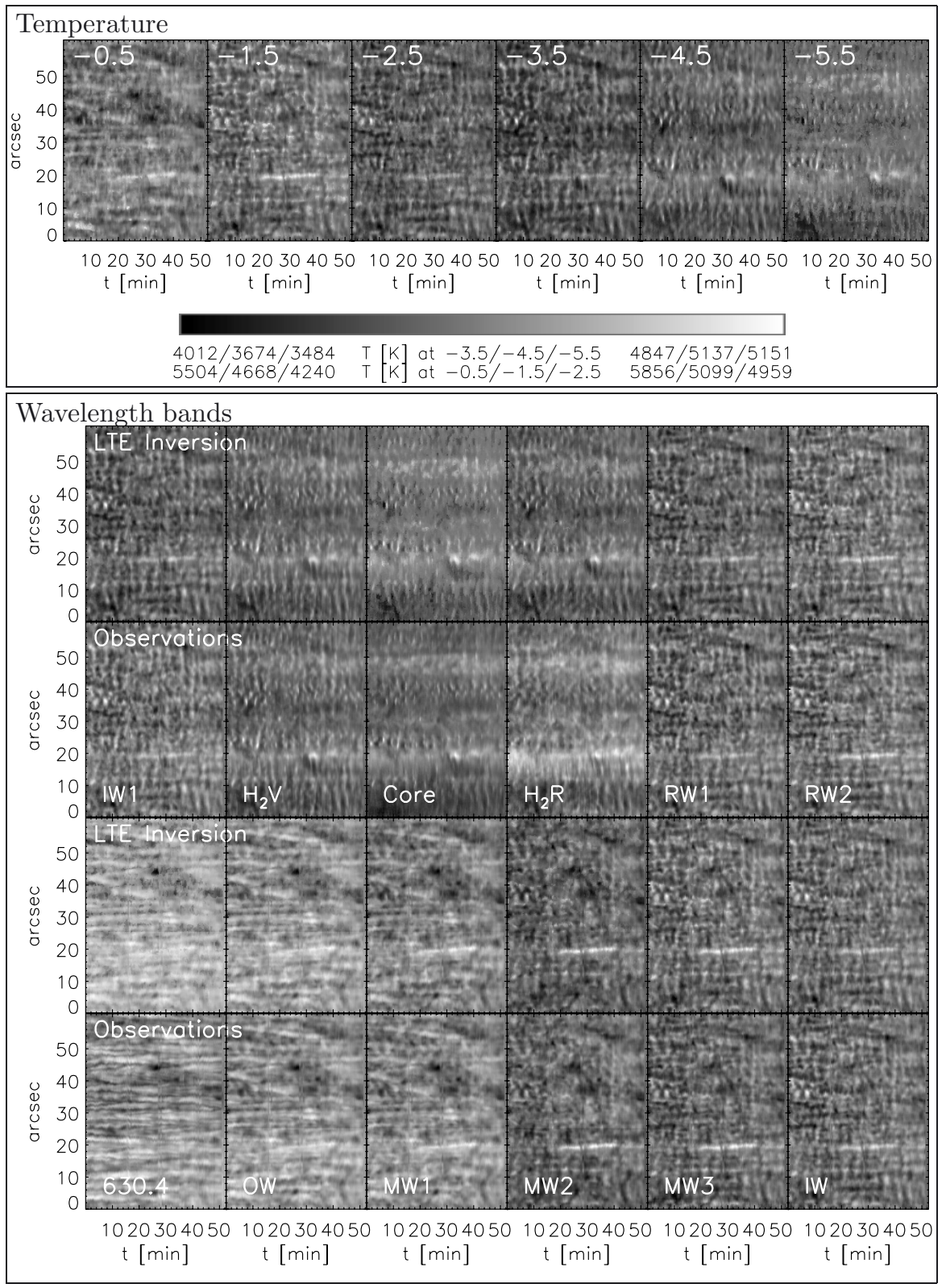

Fig. 9. Bottom panel: spatio-temporal maps of wavelengths bands in the observed spectra (odd rows) and in the best-fit spectra (even rows) of the time series. The lower two rows show from left to right the continuum intensity at $630.4 \mathrm{~nm}$, OW, MW1, MW2, MW3, and IW. The upper two rows show $\mathrm{IW} 1, \mathrm{H}_{2 \mathrm{~V}}$, core intensity, $\mathrm{H}_{2 \mathrm{R}}$, RW1, and RW2. Top panel: corresponding temperature maps at several optical depths from -0.5 to -5.5 in $\log \tau$ (denoted at the top in each subpanel). The minimum and maximum temperatures are denoted at the left and right ends of the grey bar, respectively. The three values on each side in the lower row correspond to the layers of $\log \tau=-0.5 /-1.5 /-2.5$ (from left to right), those in the upper row to $\log \tau=-3.5 /-4.5 /-5.5$. at $(x, y) \sim\left(40^{\prime \prime}, 10^{\prime \prime}-20^{\prime \prime}\right)$. The increase in emission between the two sunspots at $\log \tau=-3$ to -5 could indicate the presence of a current sheet that separates their field lines, similar to the current sheet discussed in Tritschler et al. (2008) for Ca II IR observations (see also Goodman \& Judge 2012).

\section{Discussion}

\subsection{Quality in reproducing observed spectra}

The comparison of the 2D intensity maps of observed and bestfit spectra showed some differences between the two. To quantify the resulting mismatch, we made scatter plots of the observed and best-fit intensities in the wavelength bands for the large-area scan at disc centre (Fig. 12). The graphs confirm that the intensity in the continuum at $630.4 \mathrm{~nm}$ and in the $\mathrm{H}_{2 \mathrm{R}}$ emission peak deviate between observations and best-fit spectra: for the former, the slope differs from unity, while for the latter the scatter is significantly larger than in, e.g., $\mathrm{H}_{2 \mathrm{~V}}$. For all other wavelength bands the $3-\sigma$ interval of scatter (red lines) covers the line of the one-to-one correlation. The bottom right-hand panel of Fig. 12 shows the standard deviation of the difference between the observed and best-fit intensities as a function of wavelength. Besides the location of the line blends, the rms deviation is about $0.5 \%$ of $I_{\mathrm{c}}$ in the line wing, and increases to about $1 \%$ of $I_{\mathrm{c}}$ for wavelengths in the line core from 396.82 to $396.88 \mathrm{~nm}$. The rms at the location of the $\mathrm{H}_{2 \mathrm{R}}$ emission peak is slightly enhanced relative to the rest of the line core.

The spatial variation in the reproduction of the observed spectra is displayed in Fig. 13. In the map of $\chi^{2}$ with equal weighting for all wavelengths, i.e., $w(\lambda) \equiv 1$, that measures the quality of the fit to the full line profile (top right panel), the centres of the network regions stand out, but only slightly. The cellular granular-sized pattern in the $\chi^{2}$-map corresponds to spatial structures seen in wavelengths forming at and below the MW and indicates misfits in the lower atmosphere. Calculating the $\chi^{2}$ with the enhanced weighting for the line core as used in retrieving the best-fit archive profile (bottom left panel) yields a similar spatial variation of the fit quality, with the network locations now standing out more prominently and a more homogeneous quality throughout the inter-network regions. The marker 

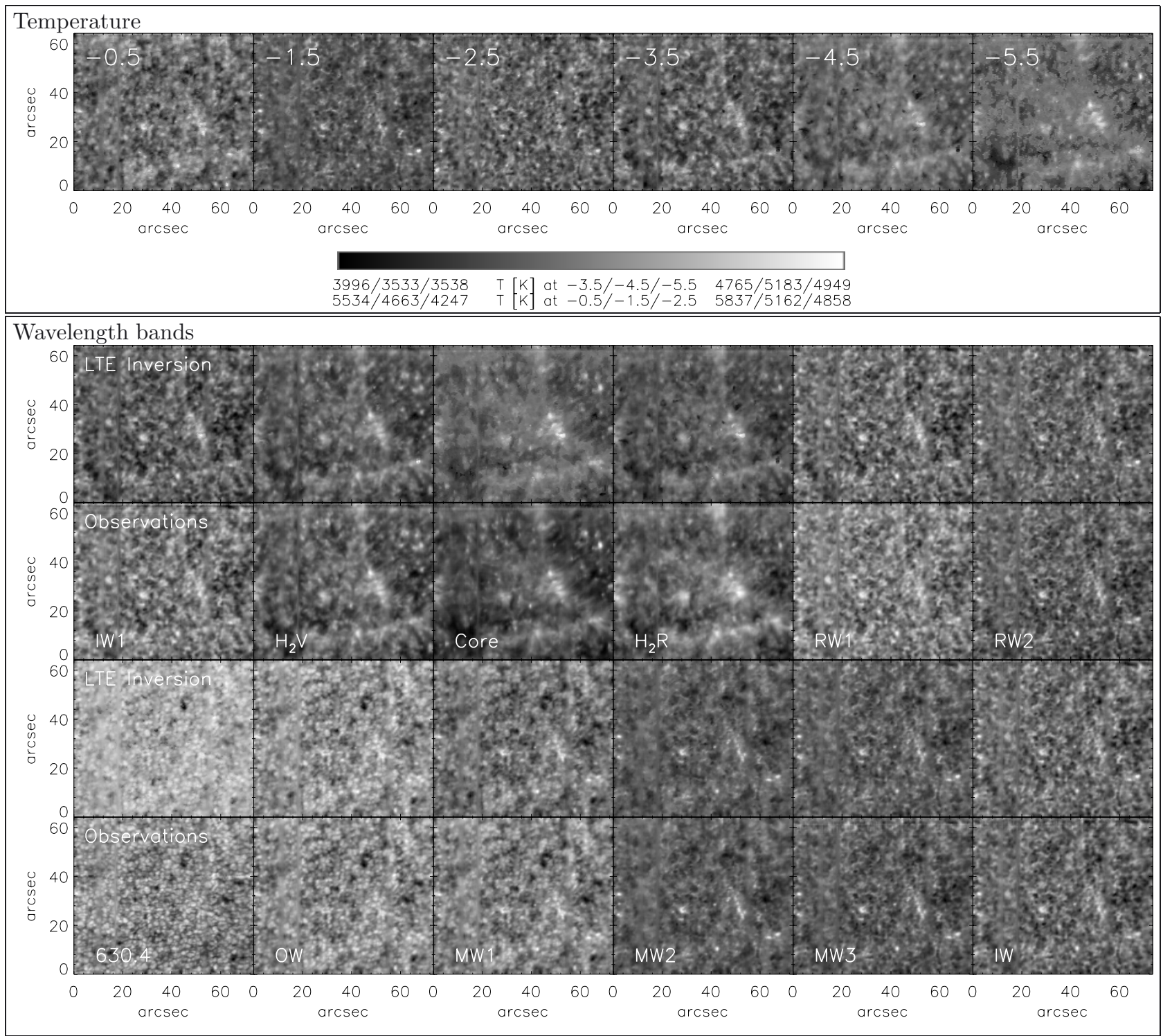

Fig. 10. Bottom panel: 2D maps of wavelengths bands in the observed spectra (odd rows) and in the best-fit spectra (even rows) of the large-area scan on disc centre. The lower two rows show from left to right the continuum intensity at $630.4 \mathrm{~nm}$, OW, MW1, MW2, MW3, and IW. The upper two rows show IW $1, \mathrm{H}_{2 \mathrm{~V}}$, the line-core intensity, $\mathrm{H}_{2 \mathrm{R}}$, RW1, and RW2. Top panel: corresponding temperature maps at several optical depths from -0.5 to -5.5 in $\log \tau$ (denoted at the top in each subpanel). See Fig. 9 for additional information on the grey bar.

that shows how the $\chi^{2}$ with the enhanced weighting for the line core was modified by the iterative improvement of the fit is displayed in the lower right panel of Fig. 13. It turns out that on about $60 \%$ of the FOV, the iterative modification of the full temperature stratification has worsened the initial fit to the line core obtained using only the best-fit archive profile. In only the internetwork regions with usually less complex profile shapes, the iteration has improved the fit not only to the line wing, but also to the line core even with the strongly enhanced contribution of the line core. For the AR data, in nearly all cases (>90\%) the fit to the line core was worsened in the iterative modification. We note that this is a slight worsening relative to the best-fit archive profile, with a still acceptable fit to the line shape even for most of the AR spectra (e.g., Fig. 8).

\subsection{Statistics on the archive usage}

The size of the pre-calculated spectral archive partly limits the usefulness of the two-step inversion approach described here. At the spectral sampling of the POLIS data of $1.92 \mathrm{pm}$ per pixel and the total wavelength range covered, the full archive of about 300000 spectra could be easily kept in the memory of a simple standard desktop machine because its size was only about $350 \mathrm{MB}$. Figure 14 shows the statistics on how often which archive profile was actually used in the determination of the best-fit archive profile. The most frequently used archive profiles appear with about $3-4 \%$ relative frequency. Some areas in the archive were only relevant in either the QS or the AR data, e.g., the archive profiles with numbers above 200000 only appear for the AR spectra. These profiles correspond to a large positive offset $T_{\text {diff }}$ in temperature in their synthesis. This global offset in temperature between the QS and AR data is caused by both the change in the heliocentric angle, where in the AR data the LOS reaches a given optical depth at a different geometrical height in comparison to the QS data, and the presence of magnetic fields throughout nearly all of the FOV in the AR that is known to lead to increased emission in Ca spectra (Rezaei et al. 2007; Rezaei 2008). 
C. Beck et al.: The energy of waves: LTE inversion of Ca II H spectra

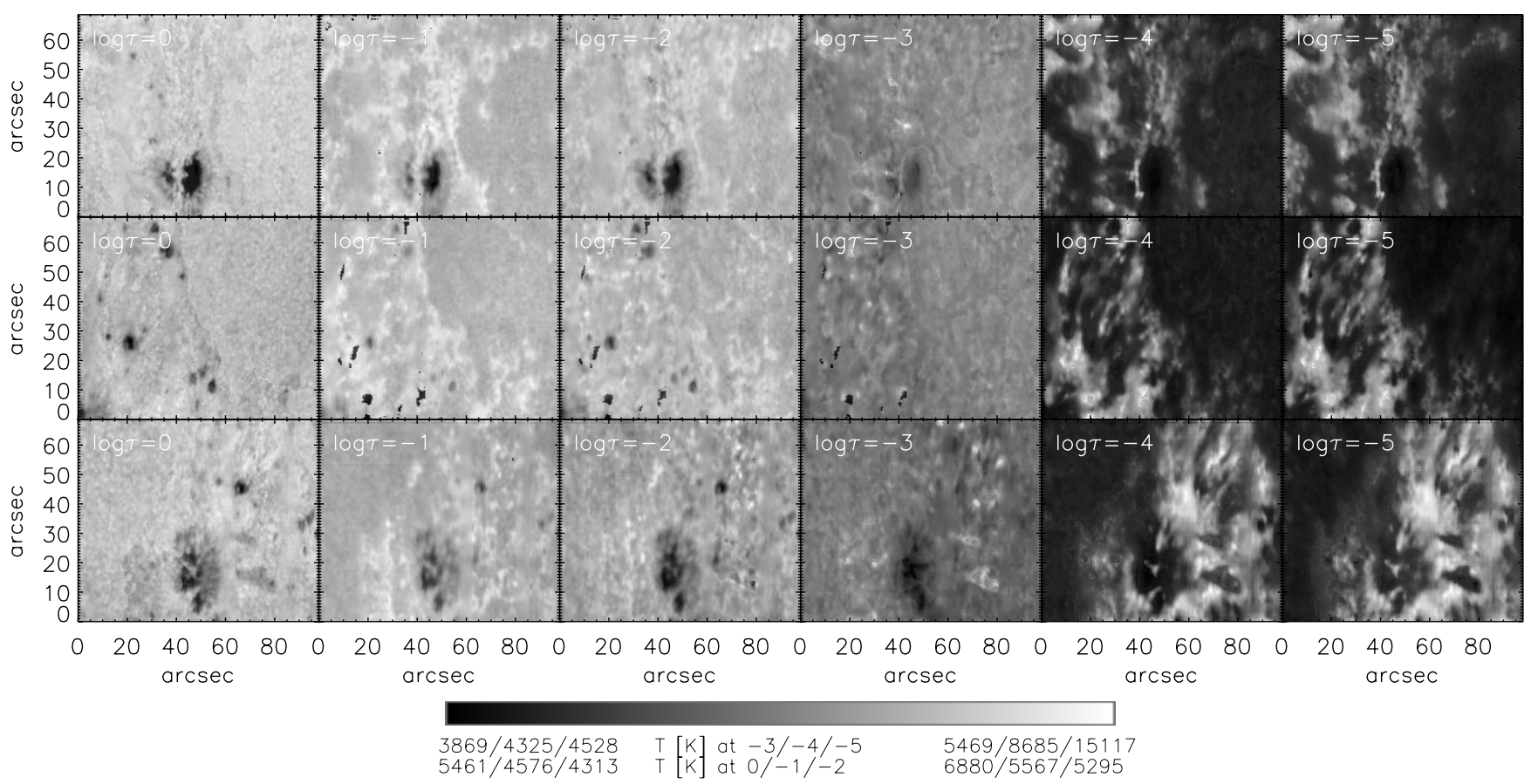

Fig. 11. Temperature maps for the active region scans. The layers of $\log \tau$ differ slightly $(0$ to -5 in $\log \tau)$. The temperature values of the grey bar refer to the middle row as reference, the range in the other two scans is of comparable order.
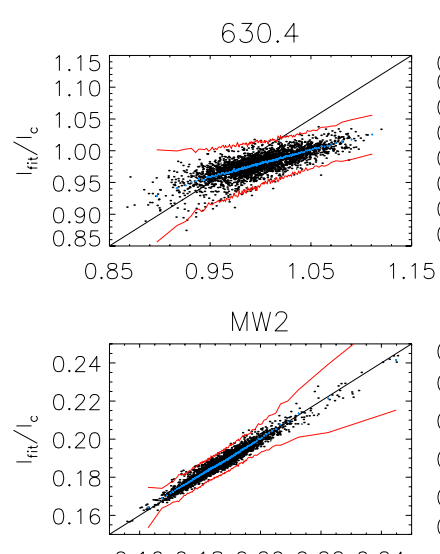

$\begin{array}{lllll}0.16 & 0.18 & 0.20 & 0.22 & 0.24\end{array}$

IW 1
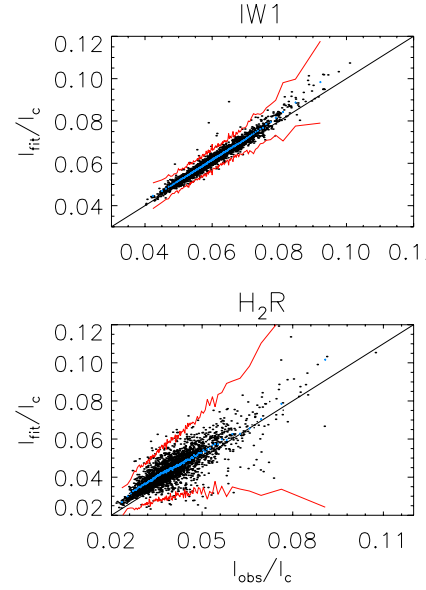

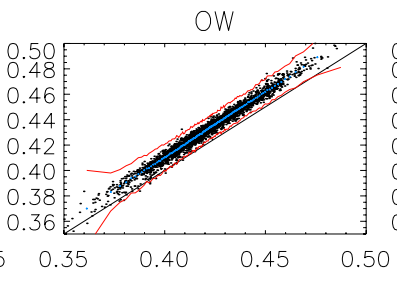

MW3

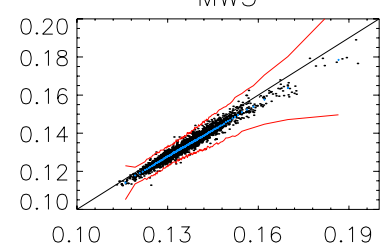

$\mathrm{H}_{2} \mathrm{~V}$

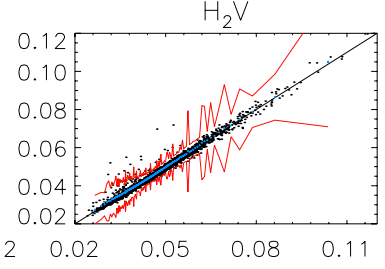

RW

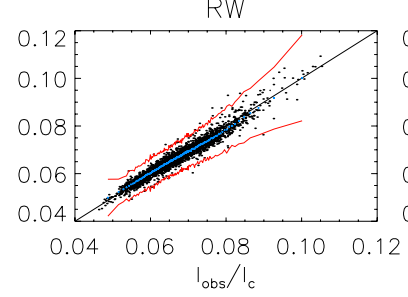

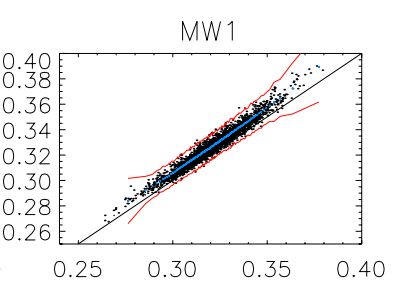

IW

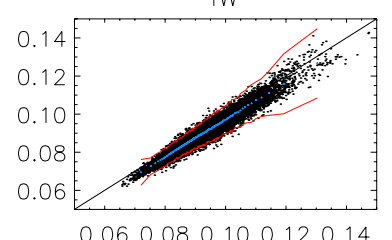

Core
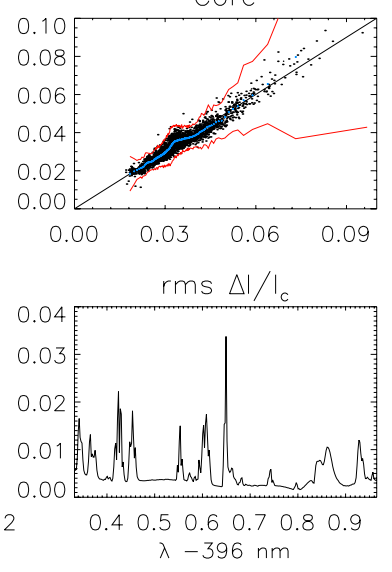

Fig. 12. Scatter plots of observed and best-fit intensities (black dots) of the large-area scan on disc centre. The blue dots denote binned values whose $3-\sigma$ variation is denoted by the red lines. The solid black line denotes a one-to-one correlation. Bottom right panel: standard deviation of the difference between observed and best-fit intensity as a function of wavelength.
It turns out that the actual usage of the archive is more sparse than the plot of Fig. 14 suggests. In total, only about 5700 different archive profiles ( $\sim 2 \%$ of the full archive) were picked in the inversion of either QS or AR data ( $~ 80000$ observed spectra). The acceptable quality of the fits in both QS and AR implies that an exchange of the LTE archive for about 10000 profiles calculated in NLTE conditions could already suffice to provide a reasonable NLTE fit to observed chromospheric Ca II H profiles, hence also to similar spectra in other chromospheric lines. The full dynamical range of the temperature variations seems to be 


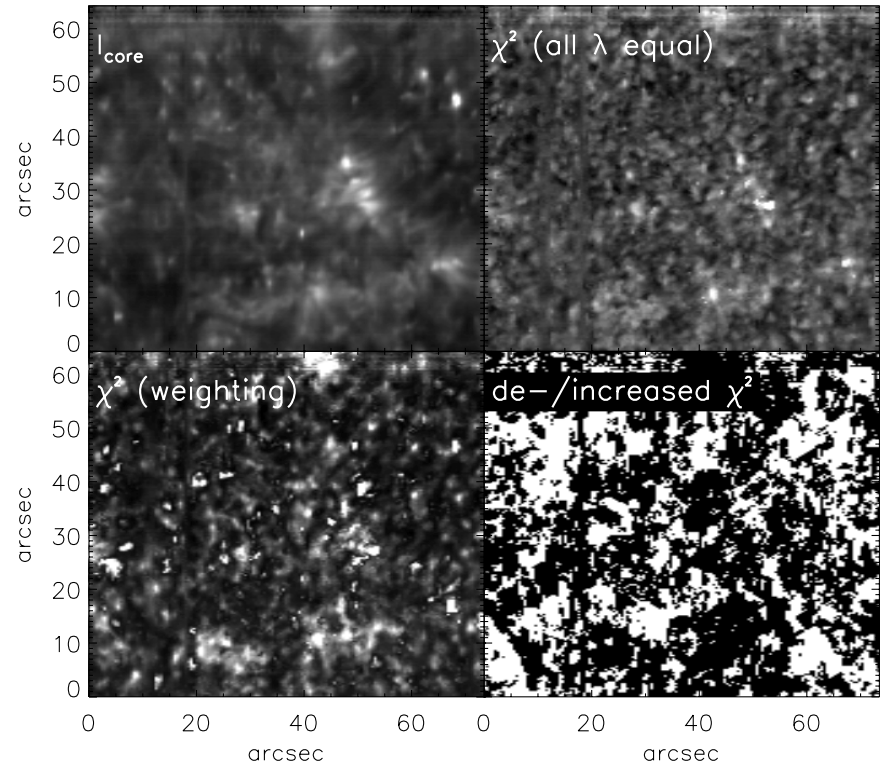

Fig. 13. Spatial variation of the $\chi^{2}$ in the large-area QS scan. Top row, left: line-core intensity as indicator of the solar spatial structuring. Top right: $\chi^{2}$ with equal weight for all wavelengths. Bottom left: $\chi^{2}$ with enhanced weighting for the line core. Bottom right: decrease/increase in $\chi^{2}$ (white/black) by the iterative modification of the fit when considering enhanced weighting in the line core.

more critical in creating the spectral archive than the density of the sampling inside the parameter space of temperature.

\subsection{Outlook}

Our findings can be extended to what will be needed for the future analysis of spectra from, e.g., the planned Solar-C mission, regardless of a decision for the $\mathrm{Ca}$ II $\mathrm{H}$ and $\mathrm{K}$ or the $\mathrm{Mg}$ II $\mathrm{h}$ and $\mathrm{k}$ lines (Katsukawa et al. 2011; Belluzzi \& Trujillo Bueno 2012) because of their similar behaviour, or data from the planned BLue Imaging Solar Spectrometer (BLISS, Puschmann et al. 2012b) for the new 1.5-m GREGOR telescope (e.g., Schmidt et al. 2012). The BLISS data will be accompanied by spectropolarimetric observations at, e.g., $630 \mathrm{~nm}$ with the GREGOR Fabry-Perót Interferometer (Puschmann et al. 2012a-c), which will provide the possibility to simultaneously measure photospheric magnetic fields as in the case of POLIS.

For deriving the thermodynamic properties of the solar chromosphere from observed spectra, direct analysis tools will be few and very limited. The best approach seems to consist in a "controlled" NLTE forward modelling, i.e., a creation of NLTE spectra for what can be considered realistic solar atmosphere models. These could be derived from both analytical 2D solutions of solar surface structures (Uitenbroek 2011) or synthetic spectra from numerical simulations (Leenaarts et al. 2009, 2010; Wedemeyer-Böhm \& Carlsson 2011). The lateral radiative transport is more important for chromospheric than for photospheric lines. A creation of an archive from 3D numerical simulations might thus be anyway required for a consistent spectral synthesis (cf. Uitenbroek \& Criscuoli 2011; Sheminova 2012; Leenaarts et al. 2012). Such an archive then also allows one to improve the inversion procedure beyond the common single-pixel approach in which every pixel is inverted individually neglecting its surroundings. Especially for chromospheric lines, a simultaneous fit to a small-scale area of, e.g., about $1^{\prime \prime} \times 1^{\prime \prime}(3 \times 3$ pixels at the POLIS spatial sampling) can be crucial for consistent results. An

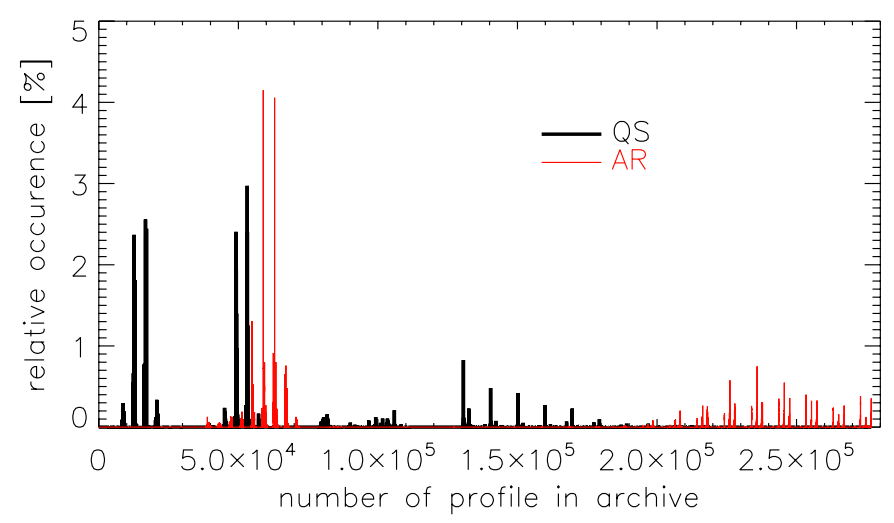

Fig. 14. Relative occurrence of a given archive profile in the inversion. Thick black: QS map. Thin red: AR map.

NLTE archive from 3D numerical simulations can directly provide suited spectra on a 2D area including a central pixel and its surroundings. There will be a need for additional modification of initial profiles and the related thermodynamical variables to match the observed spectra, similar to the two-stage approach taken here, because the combined parameter space of the uncorrelated temperature and velocity variations cannot be covered by any reasonably sized archive.

The creation of a base of NLTE spectra seems to be a promising approach for both the $\mathrm{Ca}$ and $\mathrm{Mg}$ lines, with the advantage that such spectra can already be created and tested against simulations and/or theoretical models (cf. de la Cruz Rodríguez et al. 2012) well in advance of the real data to be obtained in a few years.

The results of the retrieved temperature stratification in QS and AR will be discussed in the subsequent paper of this series.

\section{Conclusion}

A pre-calculated archive of about 300000 spectra is found to be more than sufficient to fit the line cores of observed Ca II H profiles in the quiet Sun and active regions satisfactorily regardless of the huge range of possible shapes, whereas the line wing has to be iteratively modified, because the line core and line wing vary independently of each other. The advantage of the two-step inversion approach is that an exchange of the archive for a suitable data base of spectra calculated in NLTE suffices to provide a first-order NLTE inversion method.

Cram (1977) stated that "the properties of the solar chromospheric plasma are so poorly known that all careful studies would be enlightening, no matter how crude". We have to leave it up to the reader to decide whether we have met the requirement to provide a "careful study". By using the LTE assumption, we are, however, perfectly convinced that we do fulfil his labelling of a "crude" method.

Acknowledgements. The VTT is operated by the Kiepenheuer-Institut für Sonnenphysik (KIS) at the Spanish Observatorio del Teide of the Instituto de Astrofísica de Canarias (IAC). The POLIS instrument has been a joint development of the High Altitude Observatory (Boulder, USA) and the KIS. C.B. acknowledges partial support by the Spanish Ministry of Science and Innovation through project AYA2010-18029 and JCI-2009-04504. R.R. acknowledges financial support by the DFG grant RE 3282/1-1. We thank M. Collados, H. Balthasar, J. Staude, and H. Socas-Navarro for their comments on the manuscript. 


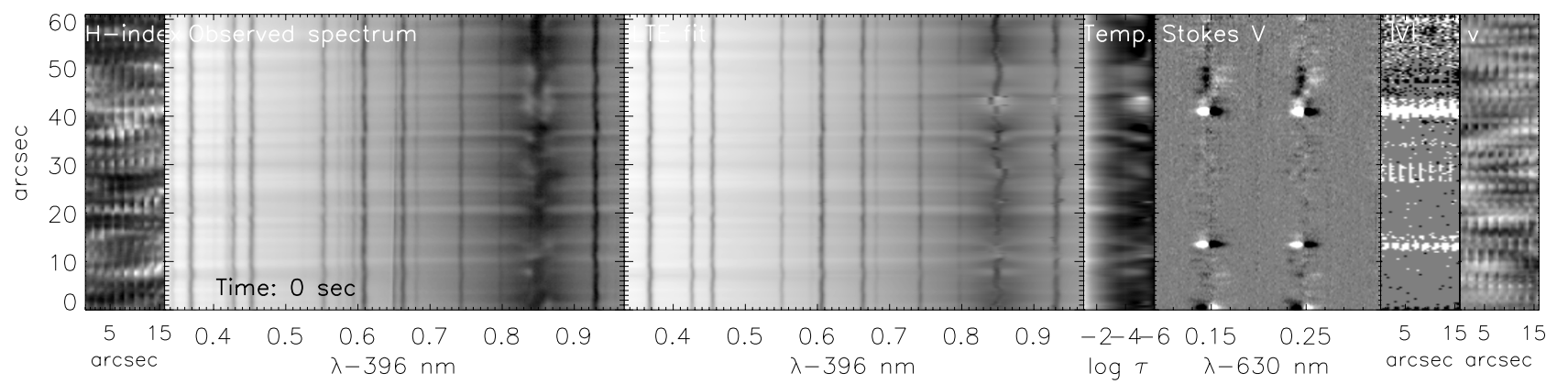

Fig. A.1. Still from an animation of the inversion results for the time series of the quiet Sun. Leftmost panel: 2D maps of the H-index for eight repetitions scanning the same area of $2^{\prime \prime} \times 61^{\prime \prime}$. Time increases left to right. The scan step of the spectra shown in the other panels is always located at the very left border at $0^{\prime \prime}$. Second and third panels: observed and best-fit Ca spectra. Fourth panel: temperature stratifications along the slit corresponding to the best-fit spectra. Fifth panel: co-spatial and simultaneous Stokes $V$ spectra at $630 \mathrm{~nm}$. Sixth panel: 2D maps of the polarity of the Stokes $V$ signal for eight repetitions scanning the same area. Seventh panel: 2D maps of the LOS velocity of the Fe I line at $630.15 \mathrm{~nm}$ for eight repetitions of scanning the same area.

\section{Appendix A: Animation of inversion results}

Figure A.1 shows a still from an animation that displays the inversion results and some line characteristics from both the $\mathrm{Ca}$ and the $630 \mathrm{~nm}$ spectra for the time series of the quiet Sun. There are three 2D maps: the H-index (integration of the line-core intensity of $\mathrm{Ca}$ ) in the first panel, the polarity of the Stokes $V$ signal ( \pm 1 for magnetic fields along/anti-parallel to the LOS and zero for locations without significant polarisation signal) in the sixth panel, and the LOS velocity of the Fe I line at $630.15 \mathrm{~nm}$ in the seventh panel. For all these 2D maps, eight repetitions of scanning the same area of $2^{\prime \prime} \times 61^{\prime \prime}$ are shown, i.e., the temporal evolution inside the area over the course of about 170 seconds is displayed. The spectra shown in the rest of the panels were always taken from the scan step that is located at $0^{\prime \prime}$ in the $2 \mathrm{D}$ maps. The animation runs over the complete duration of the time series of about one hour.

In the 2D maps of the H-index, the lateral spatial motion of individual bright grains can be followed. The temporal evolution of the temperature stratifications shows the spatial (along the slit and in height) and temporal extent of wave trains and temperature perturbations. One prominent example of an upwards propagating temperature perturbation that subsequently affects the full line profile appears between $t=378$ and $483 \mathrm{~s}$ at $y \sim 28^{\prime \prime}$, i.e., at about the middle of the FOV along the slit. Lateral motion of temperature perturbations along the slit can be seen for some events that happen close to locations with a strong polarisation signal. One such event shows up between $t=693$ and 756 seconds, starting at the location of the persistent polarisation signal at $y \sim 40^{\prime \prime}$ and moving downwards along the slit with time, while getting increasingly weaker on the way. The Stokes $V$ spectra and the polarity of the Stokes $V$ signal show that most of the polarisation signals, hence detected photospheric magnetic fields, are fairly stable throughout the time series. With an integration time of less than four seconds per scan step, the polarimetric sensitivity in the time series is naturally limited to strong polarisation signals that correspond to the stable photospheric magnetic network.

The polarity was erroneously labelled with " $|V|$ " during the creation of the animation.

\section{References}

Balasubramaniam, K. S., Christopoulou, E. B., \& Uitenbroek, H. 2004, ApJ, 606,1233

Beck, C. 2011, A\&A, 525, A133

Beck, C. A. R., \& Rammacher, W. 2010, A\&A, 510, A66
Beck, C., Schmidt, W., Kentischer, T., \& Elmore, D. 2005, A\&A, 437, 1159 Beck, C., Collados, M. V., Khomenko, E., \& Rezaei, R. 2008a, 12th Europ. Solar Physics Meet., Freiburg, Germany, September 8-12, Session 2.2-11

Beck, C., Schmidt, W., Rezaei, R., \& Rammacher, W. 2008b, A\&A, 479, 213 (BE08)

Beck, C., Khomenko, E., Rezaei, R., \& Collados, M. 2009, A\&A, 507, 453 (BE09)

Beck, C., Tritschler, A., \& Wöger, F. 2010, Astron. Nachr., 331, 574

Beck, C., Rezaei, R., \& Fabbian, D. 2011, A\&A, 535, A129 (BE11)

Beck, C., Rezaei, R., \& Puschmann, K. G. 2012, A\&A, 544, A46 (BE12)

Beckers, J. M. 1968, Sol. Phys., 3, 367

Belluzzi, L., \& Trujillo Bueno, J. 2012, ApJ, 750, L11

Berlicki, A., Heinzel, P., Schmieder, B., Mein, P., \& Mein, N. 2005, A\&A, 430, 679

Bethge, C., Beck, C., Peter, H., \& Lagg, A. 2012, A\&A, 537, A130

Bonnet, R. M. 1981, Space Sci. Rev., 29, 131

Bostanc1, Z. F. 2011, Astron. Nachr., 332, 815

Bray, R. J. 1973, Sol. Phys., 29, 317

Carlsson, M., \& Stein, R. F. 1997, ApJ, 481, 500

Carlsson, M., \& Stein, R. F. 2002, ApJ, 572, 626

Carlsson, M., Judge, P. G., \& Wilhelm, K. 1997, ApJ, 486, L63

Cauzzi, G., Reardon, K. P., Uitenbroek, H., et al. 2008, A\&A, 480, 515

Cauzzi, G., Reardon, K., Rutten, R. J., Tritschler, A., \& Uitenbroek, H. 2009, A\&A, 503, 577

Collados, M., Lagg, A., Díaz García, J. J., et al. 2007, in The Physics of Chromospheric Plasmas, eds. P. Heinzel, I. Dorotovič, \& R. J. Rutten, ASP Conf. Ser., 368, 611

Cram, L. E. 1977, A\&A, 59, 151

Cram, L. E., \& Dame, L. 1983, ApJ, 272, 355

Curdt, W., Tian, H., Teriaca, L., \& Schühle, U. 2010, A\&A, 511, L4

de la Cruz Rodríguez, J., Socas-Navarro, H., Carlsson, M., \& Leenaarts, J. 2012, A\&A, 543, A34

Delbouille, L., Roland, G., \& Neven, L. 1973, Atlas photometrique du spectre solaire de $\lambda=3000 \AA$ a $10000 \AA$ (Liège: Universite de Liège, Institut d'Astrophysique)

Felipe, T., Khomenko, E., Collados, M., \& Beck, C. 2010, ApJ, 722, 131

Fontenla, J., Reichmann, E. J., \& Tandberg-Hanssen, E. 1988, ApJ, 329, 464

Fontenla, J. M., Avrett, E., Thuillier, G., \& Harder, J. 2006, ApJ, 639, 441

Gingerich, O., Noyes, R. W., Kalkofen, W., \& Cuny, Y. 1971, Sol. Phys., 18, 347

Goodman, M. L., \& Judge, P. G. 2012, ApJ, 751, 75

Grigoryeva, S. A., Turova, I. P., \& Teplitskaia, R. B. 1991, Sol. Phys., 135, 1

Grigoryeva, S. A., Ozhogina, O. A., \& Teplitskaya, R. B. 2000, Sol. Phys., 195, 269

Grigoryeva, S. A., Teplitskaya, R. B., \& Ozhogina, O. A. 2009, Astron. Rep., 53, 569

Holweger, H., \& Müller, E. A. 1974, Sol. Phys., 39, 19

Jensen, E., \& Orrall, F. Q. 1963, ApJ, 138, 252

Katsukawa, Y., Suematsu, Y., Shimizu, T., Ichimoto, K., \& Takeyama, N. 2011, in Solar Physics and Space Weather Instrumentation, eds. S. Fineschi, \& J. Fenelly, SPIE Conf. Ser., 8148

Kneer, F., \& Mattig, W. 1978, A\&A, 65, 17

Kneer, F., Mattig, W., \& v. Uexkuell, M. 1981, A\&A, 102, 147

Kuckein, C., Centeno, R., Martínez Pillet, V., et al. 2009, A\&A, 501, 1113

López Ariste, A., Socas-Navarro, H., \& Molodij, G. 2001, ApJ, 552, 871

Leenaarts, J., Carlsson, M., Hansteen, V., \& Rouppe van der Voort, L. 2009, ApJ, 694, L128 
Leenaarts, J., Rutten, R. J., Reardon, K., Carlsson, M., \& Hansteen, V. 2010, ApJ, 709, 1362

Leenaarts, J., Carlsson, M., \& Rouppe van der Voort, L. 2012, ApJ, 749, 136

Linsky, J. L., \& Avrett, E. H. 1970, PASP, 82, 169

Lites, B. W. 1986, ApJ, 301, 1005

Lites, B. W., \& Skumanich, A. 1982, ApJS, 49, 293

Lites, B. W., Rutten, R. J., \& Berger, T. E. 1999, ApJ, 517, 1013

Liu, S.-Y. 1974, ApJ, 189, 359

Liu, S.-Y., \& Skumanich, A. 1974, Sol. Phys., 38, 109

López Ariste, A., Casini, R., Paletou, F., et al. 2005, ApJ, 621, L145

Martínez Pillet, V., Collados, M., Sánchez Almeida, J., et al. 1999, in High Resolution Solar Physics: Theory, Observations, and Techniques, ASP Conf. Ser., 183, 264

Martínez-Sykora, J., De Pontieu, B., \& Hansteen, V. 2012, ApJ, 753, 161

Molowny-Horas, R., Heinzel, P., Mein, P., \& Mein, N. 1999, A\&A, 345, 618

Pietarila, A., Socas-Navarro, H., \& Bogdan, T. 2007a, ApJ, 670, 885

Pietarila, A., Socas-Navarro, H., \& Bogdan, T. 2007b, ApJ, 663, 1386

Puschmann, K. G., \& Beck, C. 2011, A\&A, 533, A21

Puschmann, K. G., Ruiz Cobo, B., Vázquez, M., Bonet, J. A., \& Hanslmeier, A 2005, A\&A, 441, 1157

Puschmann, K. G., Ruiz Cobo, B., \& Martínez Pillet, V. 2010a, ApJ, 720, 1417

Puschmann, K. G., Ruiz Cobo, B., \& Martínez Pillet, V. 2010b, ApJ, 721, L58

Puschmann, K. G., Balthasar, H., Bauer, S.-M., et al. 2012a, in Magnetic fields from the photosphere to the corona, eds. T. R. Rimmele, A. Tritschler, F. Woeger, et al., ASP Conf. Ser., 463, 423

Puschmann, K. G., Balthasar, H., Beck, C., et al. 2012b, in Ground-based and Airborne Instrumentation for Astronomy IV, eds. I. S. MacLean, S. K. Ramsay, \& H. Takami, SPIE Conf. Ser., 8446, 844679

Puschmann, K. G., Denker, C., Kneer, F., et al. 2012c, AN, 333, 880

Rammacher, W., \& Cuntz, M. 2005, A\&A, 438, 721

Rammacher, W., \& Ulmschneider, P. 1992, A\&A, 253, 586

Rezaei, R. 2008, Ph.D. Thesis, Albert-Ludwigs University, Freiburg

Rezaei, R., Schlichenmaier, R., Beck, C. A. R., Bruls, J. H. M. J., \& Schmidt, W. 2007, A\&A, 466, 1131

Rezaei, R., Bruls, J. H. M. J., Schmidt, W., et al. 2008, A\&A, 484, 503

Rouppe van der Voort, L. H. M. 2002, A\&A, 389, 1020

Rüedi, I., Solanki, S. K., \& Livingston, W. C. 1995, A\&A, 293, 252

Ruiz Cobo, B., \& del Toro Iniesta, J. C. 1992, ApJ, 398, 375

Ruiz Cobo, B., \& Puschmann, K. G. 2012, ApJ, 745, 141

Rutten, R. J., \& Uitenbroek, H. 1991, Sol. Phys., 134, 15

Sánchez Almeida, J., \& Lites, B. W. 2000, ApJ, 532, 1215

Sánchez-Andrade Nuño, B., Centeno, R., Puschmann, K. G., et al. 2007, A\&A, 472, L51
Sánchez-Andrade Nuño, B., Bello González, N., Blanco Rodríguez, J., Kneer, F., \& Puschmann, K. G. 2008, A\&A, 486, 577

Schmidt, W., von der Lühe, O., Volkmer, R., et al. 2012, in The GREGOR solar telescope on Tenerife, ASP Conf. Ser., in press [arXiv: 1202.4289]

Schmieder, B., Mein, P., Martres, M. J., \& Tandberg-Hanssen, E. 1984, Sol. Phys., 94, 133

Schmieder, B., Tziotziou, K., \& Heinzel, P. 2003, A\&A, 401, 361

Schröter, E. H., Soltau, D., \& Wiehr, E. 1985, Vist. Astron., 28, 519

Sheminova, V. A. 2012, Sol. Phys., 280, 83

Sheminova, V. A., Rutten, R. J., \& Rouppe van der Voort, L. H. M. 2005, A\&A, 437, 1069

Shine, R. A., \& Linsky, J. L. 1974a, Sol. Phys., 37, 145

Shine, R. A., \& Linsky, J. L. 1974b, Sol. Phys., 39, 49

Socas-Navarro, H. 2005, ApJ, 631, L167

Socas-Navarro, H., \& Elmore, D. 2005, ApJ, 619, L195

Socas-Navarro, H., Ruiz Cobo, B., \& Trujillo Bueno, J. 1998, ApJ, 507, 470

Socas-Navarro, H., Trujillo Bueno, J., \& Ruiz Cobo, B. 2000, ApJ, 530, 977

Socas-Navarro, H., López Ariste, A., \& Lites, B. W. 2001, ApJ, 553, 949

Socas-Navarro, H., Martínez Pillet, V., Elmore, D., et al. 2006, Sol. Phys., 235, 75

Solanki, S. K., Steiner, O., \& Uitenbroek, H. 1991, A\&A, 250, 220 (SO91)

Suemoto, Z. 1977, Sol. Phys., 54, 3

Teplitskaja, R. B., \& Efendieva, S. A. 1975, Sol. Phys., 43, 293

Teplitskaia, R. B., \& Firstova, N. M. 1976, Sol. Phys., 48, 103

Teplitskaya, R. B., \& Grigoryeva, S. A. 2009, Geomagnetism and Aeronomy/ Geomagnetizm i Aeronomiia, 49, 916

Teplitskaya, R. B., Turova, I. P., \& Ozhogina, O. A. 2009, Astron. Lett., 35, 712

Tritschler, A., Uitenbroek, H., \& Reardon, K. 2008, ApJ, 686, L45

Tsiropoula, G., Alissandrakis, C. E., \& Schmieder, B. 1993, A\&A, 271, 574

Tziotziou, K. 2007, in The Physics of Chromospheric Plasmas, eds. P. Heinzel, I. Dorotovič, \& R. J. Rutten, ASP Conf. Ser., 368, 217

Tziotziou, K., Heinzel, P., Mein, P., \& Mein, N. 2001, A\&A, 366, 686

Uitenbroek, H. 2011, in Solar Polarization 6, eds. J. R. Kuhn, D. M. Harrington, H. Lin, et al., ASP Conf. Ser., 437, 439

Uitenbroek, H., \& Criscuoli, S. 2011, ApJ, 736, 69

Uitenbroek, H., Balasubramaniam, K. S., \& Tritschler, A. 2006, ApJ, 645, 776

Vecchio, A., Cauzzi, G., \& Reardon, K. P. 2009, A\&A, 494, 269

Vernazza, J. E., Avrett, E. H., \& Loeser, R. 1981, ApJS, 45, 635 (VAL81)

von der Lühe, O., Soltau, D., Berkefeld, T., \& Schelenz, T. 2003, in Innovative Telescopes and Instrumentation for Solar Astrophysics, eds. S. L. Keil, \& S. V. Avakyan, SPIE Conf. Ser., 4853, 187

Wedemeyer-Böhm, S., \& Carlsson, M. 2011, A\&A, 528, A1 